\title{
VLT-UVES analysis of two giants in the bulge metal-poor globular cluster HP-1 ${ }^{\star}, \star \star$
}

\section{Analysis of two giants in HP-1}

\author{
B. Barbuy ${ }^{1}$, M. Zoccali ${ }^{2}$, S. Ortolani ${ }^{3}$, Y. Momany ${ }^{5}$, D. Minniti ${ }^{1}$, V. Hill ${ }^{4}$,
} A. Renzini ${ }^{5}$, R. M. Rich ${ }^{6}$, E. Bica ${ }^{7}$, L. Pasquini ${ }^{5}$, and R. K. S. Yadav ${ }^{3,8}$

1 Universidade de São Paulo, IAG, Rua do Matão 1226, Cidade Universitária, São Paulo 05508-900, Brazil e-mail: barbuy@astro.iag.usp.br

2 Universidad Catolica de Chile, Department of Astronomy \& Astrophysics, Casilla 306, Santiago 22, Chile e-mail: [mzoccali; dante] aastro.puc.cl

3 Università di Padova, Dipartimento di Astronomia, Vicolo dell'Osservatorio 2, 35122 Padova, Italy e-mail: [ortolani; momany] @pd.astro.it

4 Observatoire de Paris-Meudon, 92195 Meudon Cedex, France e-mail: Vanessa.Hill@obspm. fr

5 European Southern Observatory, Karl Schwarzschild Strasse 2, 85748 Garching bei München, Germany e-mail: [arenzini; lpasquin]@eso.org

${ }^{6}$ UCLA, Department of Physics \& Astronomy, 8979 Math-Sciences Building, Los Angeles, CA 90095-1562, USA e-mail: rmr@astro.ucla.edu

7 Universidade Federal do Rio Grande do Sul, Departamento de Astronomia, CP 15051, Porto Alegre 91501-970, Brazil e-mail: bica@if.ufrgs.br

8 Aryabhatta Research Institute of Observational Sciences, (ARIES) Manora Peak, NainiTal 263129, India

Received 30 September 2005 / Accepted 30 November 2005

\section{ABSTRACT}

Context. Metal-poor globular clusters in the bulge are important tracers of early chemical evolution. HP-1 is among the six metal-poor clusters within $5^{\circ}$ of the Galactic center, and could be the one closest to the center

Aims. The main purpose of this study is the determination of metallicity and elemental ratios.

Methods. High resolution spectra of two giants of the bulge globular cluster HP-1 were obtained at the $8 \mathrm{~m}$ VLT UT2-Kueyen telescope with the UVES spectrograph. This is the second metal-poor globular cluster in the bulge for which a detailed abundance analysis is presented. Multiband $V, I, J, H, K_{\mathrm{s}}$ photometry was used to derive effective temperatures.

Results. The present analysis provides a metallicity $[\mathrm{Fe} / \mathrm{H}]=-1.00 \pm 0.2$. The $\alpha$-elements oxygen and silicon show $[\alpha / \mathrm{Fe}] \approx+0.3$, whereas magnesium, calcium and titanium show solar ratios. A proper motion analysis indicates that the two stars are cluster members.

Conclusions. The metallicity is unexpected for a blue Horizontal Branch (BHB) cluster. HP-1 is the first known cluster with such a high metallicity combined with a BHB and a steep Red Giant Branch (RGB). Together with NGC 6388 and NGC 6441 of $[\mathrm{Fe} / \mathrm{H}] \sim-0.6$ it would be third with such characteristics, but it differs from them, since these two other clusters have also a populous Red HB, and a normal slope of the RGB for their metallicity, which is not the case of HP-1.

Key words. Galaxy: bulge - Galaxy: globular clusters: individual: HP-1 - stars: abundances - stars: Hertzsprung-Russell (HR) and C-M diagrams

\section{Introduction}

Among the 16 known globular clusters located within $5^{\circ}$ of the Galactic center, six of them are metal-poor $([\mathrm{Fe} / \mathrm{H}] \lesssim-0.9)$,

* Observations collected both at the European Southern Observatory, Paranal and La Silla, Chile (ESO programme 65.L0340).

$\star \star$ Table 6 and Appendix A are only available in electronic form at http://www . edpsciences.org including HP-1. The characteristics of 5 of these bulge metalpoor clusters, namely Terzan 4, HP-1, NGC 6522, NGC 6540, and Terzan 10, have been reviewed by Barbuy et al. (1998), while Terzan 9 has been studied more recently by Ortolani et al. (1999). The most metal-poor cluster of these six clusters, Terzan $4([\mathrm{Fe} / \mathrm{H}]=-1.6)$, was further analysed using HST NICMOS $J, H$ photometry (Ortolani et al. 2001).

Metal-poor bulge field stars and clusters represent a crucial piece in the puzzle of the Milky Way formation. In fact, if the 
Table 1. Parameters of HP-1 given in the literature.

\begin{tabular}{|c|c|c|c|c|}
\hline$E(B-V)$ & {$[\mathrm{Fe} / \mathrm{H}]$} & $\overline{v_{\mathrm{r}}}$ & $\overline{(m-M}$ & $\overline{\text { Ref. }}$ \\
\hline 1.68 & -0.3 & $+62,-57$ & - & Minniti (1995) \\
\hline 1.44 & -0.56 & 44 & - & AZ88 \\
\hline 1.19 & -1.5 & - & 17.84 & Ortolani et al. (1997) \\
\hline 0.74 & -1.55 & 53.1 & 18.03 & Harris (1996) \\
\hline 0.74 & -1.6 & - & - & Davidge (2000) \\
\hline
\end{tabular}

Galaxy formed via dissipational collapse, then the metal-poor objects in the central region would be the first to have formed (van den Bergh 1993; Davidge 2001; Davidge et al. 2004).

The globular cluster $\mathrm{Cl}$ Haute-Provence 1 or HP-1, also designated BH 229 and ESO 455-SC11, was discovered by Dufay et al. (1954). It is located at $\alpha=17^{\mathrm{h}} 31^{\mathrm{m}} 05.2^{\mathrm{s}}, \delta=$ $-29^{\circ} 58^{\prime} 54^{\prime \prime}$ (J2000), and projected at only $3.33^{\circ}$ from the Galactic center $\left(l=357.42^{\circ}, b=2.12^{\circ}\right)$. It is at a distance $d_{\odot}=6.4 \mathrm{kpc}$ away from the Sun, and at $R_{\mathrm{GC}}=1.6 \mathrm{kpc}$ from the Galactic center (Barbuy et al. 1998). The cluster is very concentrated and has been classified as post-core collapse with a concentration parameter $c=2.50$, a core radius $r_{\mathrm{c}}=0.03^{\prime}$, a tidal radius $r_{\mathrm{t}} \approx 8.22^{\prime}$, and a half-light radius of $r_{\mathrm{h}}=3.10^{\prime}$ (Trager et al. 1995; Harris 1996).

The basic parameters of HP-1 given in the literature are gathered in Table 1. Armandroff \& Zinn (1988, hereafter AZ88) derived the cluster metallicity based on the CaII triplet, finding $[\mathrm{Fe} / \mathrm{H}]=-0.56$. Minniti (1995) measured the metallicity of HP-1 via Lick indices in 6 cluster stars, and obtained $[\mathrm{Fe} / \mathrm{H}]=-0.3$. Its metallicity was estimated to be $[\mathrm{Fe} / \mathrm{H}]=-0.5$ by Minniti et al. (1995) based on $J, K$ data and $[\mathrm{Fe} / \mathrm{H}]=-1.5$ by Ortolani et al. (1997) based on NTT $V, I$ CMDs. Based on CO indices of 3 stars Davidge (2000) suggested a metallicity of $[\mathrm{Fe} / \mathrm{H}]=-1.6$ for $\mathrm{HP}-1$. Given the large differences in $[\mathrm{Fe} / \mathrm{H}]$ presented in the literature, Bica et al. (1997) have discussed the possibility that HP-1 has captured metal-rich stars. Bica et al. (2005) confirmed that HP-1 is the cluster closest to the Galactic center.

Among the metal-poor clusters of the inner bulge, only Terzan 4 has been studied with high resolution infrared spectroscopy (Origlia \& Rich 2004), revealing significant enhancement of the $\alpha$-elements.

In this work we present detailed abundance analysis of two stars in HP-1 using high resolution échelle spectra obtained with UVES at the ESO VLT-UT2 Kueyen $8 \mathrm{~m}$ telescope. The observations are described in Sect. 2. A proper motion analysis verifying the cluster membership of the two target stars is presented in Sect. 3. Photometric stellar parameters (effective temperature and gravity) are derived in Sect. 4. Atomic and molecular data are reviewed in Sect. 5. Spectroscopic parameters are derived in Sect. 6 and abundance ratios are computed in Sect. 7. Conclusions are drawn in Sect. 7.

\section{The data}

\subsection{Imaging}

$V$ and $I$ images were collected in 1994, May 16, with the $3.55 \mathrm{~m}$ NTT telescope at the European Southern Observatory (ESO),

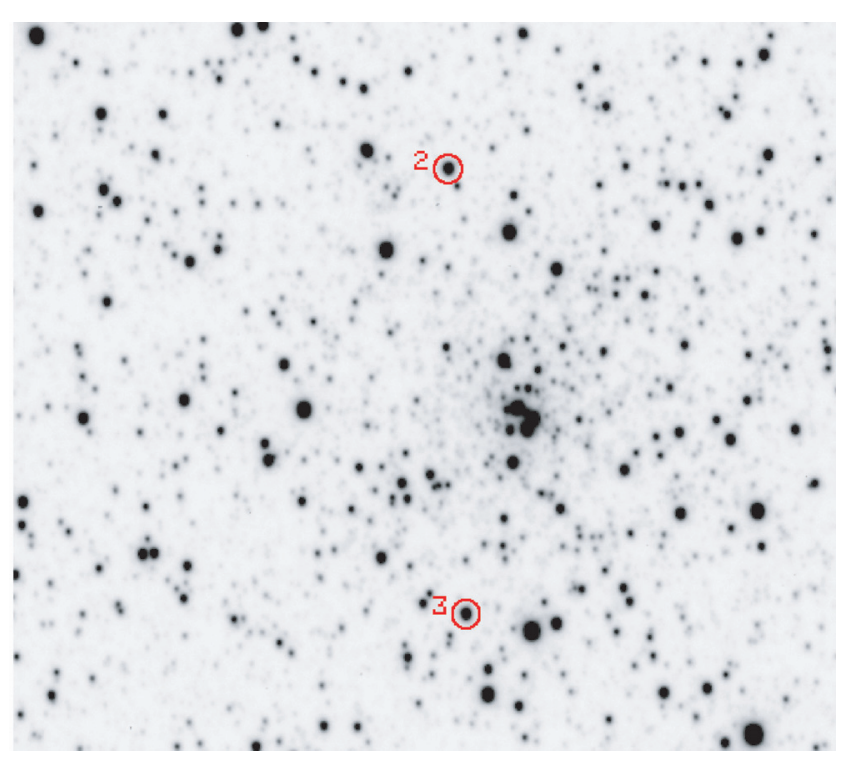

Fig. 1. Finding chart for the 2 stars observed in HP-1 (NTT-SUSI $V$ image). The frame size is $2.2 \times 2.2 \mathrm{arcmin}$, and the resolution is $0.13 \mathrm{arcsec} / \mathrm{px}$.

La Silla, Chile. The Superb Seeing Imager (SUSI) provided a field of view of $2.2 \times 2.2 \mathrm{arcmin}$, with seeing $0.45-0.6^{\prime \prime}$. For details on data reduction see Ortolani et al. (1997). The NTTSUSI $V$ image is shown in Fig. 1, where the target stars are indicated.

Another set of $B, V$ and $I$ images of HP-1 were obtained in 2002, June 19, with the Wide Field Imager (WFI) at the $2.2 \mathrm{~m}$ ESO-MPI telescope (La Silla, Chile) within our program dedicated to a large field survey of Galactic globular clusters. The WFI camera consists of eight $2048 \times 4096$ EEV-CCDs with a total field of view of $34 \times 33 \mathrm{arcmin}^{2}$. Stellar photometry was performed using the DAOPHOT II + ALLFRAME programs (Stetson 1994). The photometric calibrations were defined using standard stars from Landolt (1992). Further details on the observations and reductions can be found in Momany et al. (2002) and Alves-Brito et al. (2005).

We have tied the WFI calibration to that of NTT data, because the colour terms are more reliable in the NTT calibration. The NTT colour term in $V, I$ is of the order of $0.05 \pm 0.01 \mathrm{mag}$. This means that the correction is around $0.15 \pm 0.03 \mathrm{mag}$ at $V-I=3.0$, considerably smaller than the coefficient 0.15 and a correction of 0.45 from WFI. Furthermore the calibration equation at NTT was derived using standards up to $V-I=1.65$, while at WFI the reddest stars are around $V-I=1$.

Target stars were cross identified in the 2MASS Point Source Catalogue (Skrutskie et al. 1997), available from the WEB url: http://ipac.caltech.edu/2mass/releases/ allsky/ and their $J, H$, and $K_{\mathrm{s}}$ magnitudes are listed in Table 2.

The location of target stars on the NTT CMD is shown in Fig. 2. In this figure it is worth noting that there is a fairly well populated blue Horizontal Branch, which together with the slope of the Red Giant Branch, is suggestive of a relatively low metallicity. Figure 3 shows a larger field $(5 \times 5$ arcmin $)$ CMD extracted from the WFI photometry. The latter obviously 
Table 2. Positions and magnitudes. For each star, the first line lists the original magnitudes while the second one gives dereddened magnitudes adopting the reddening law by Dean et al. (1978) and Rieke \& Lebofsky (1985).

\begin{tabular}{ccccccccccc}
\hline \hline Star & $\alpha_{2000}$ & $\delta_{2000}$ & $E(B-V)$ & $V$ & $I$ & $J$ & $H$ & $K_{\mathrm{s}}$ & $K_{\mathrm{TCS}}$ & $K_{J}$ \\
& & & & $V_{0}$ & $I_{0}$ & $J_{0}$ & $H_{0}$ & $K_{\mathrm{s} 0}$ & $K_{\mathrm{TCS} 0}$ & $K_{J 0}$ \\
\hline HP1-2 & $17: 31: 05.852 \mathrm{M}$ & $-29: 58: 35.5$ & 1.12 & 16.982 & 14.332 & 12.21 & 11.268 & 10.969 & 10.978 & 10.997 \\
& & & & 13.510 & 12.350 & 11.224 & 10.652 & 10.570 & & \\
HP1-3 & $17: 31: 05.872 \mathrm{M}$ & \multirow{2}{*}{$-29: 59: 18.1$} & & 17.003 & 14.358 & 12.167 & 11.146 & 10.828 & 10.838 & 10.854 \\
& & & & 13.531 & 12.376 & 11.181 & 10.530 & 10.429 & & \\
\hline
\end{tabular}

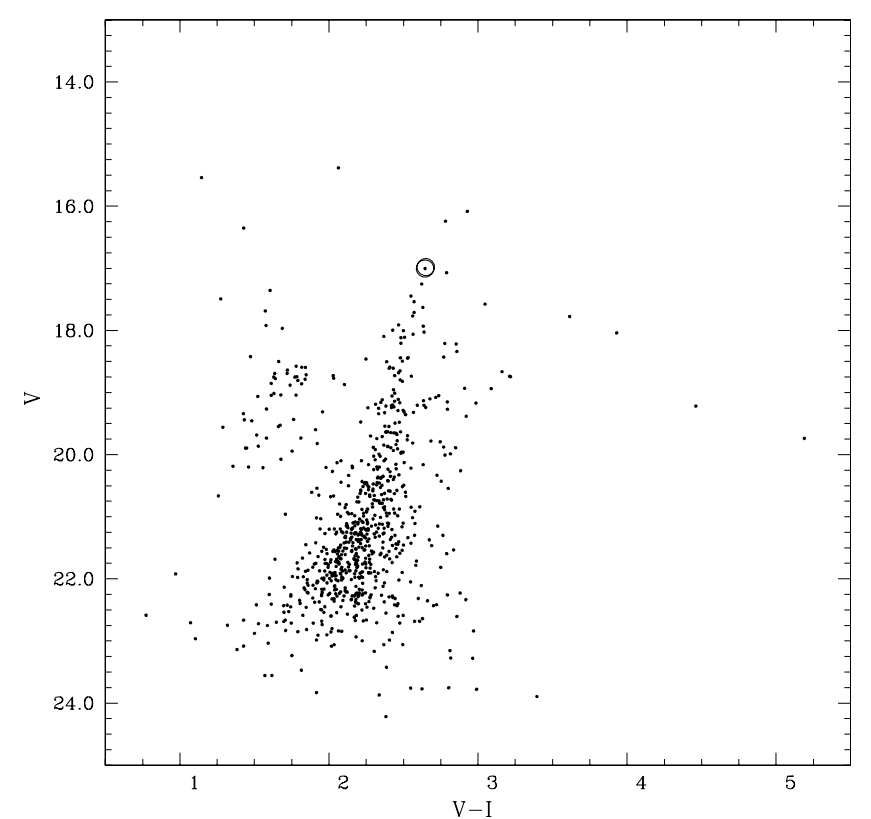

Fig. 2. The NTT-SUSI $V$ vs. $V-I$ CMD of HP-1 with the target stars (they are almost coincident) indicated.

includes many more bulge foreground stars. Although in Fig. 2 the relative contamination bulge/cluster stars is significantly lower, a metal-rich Red Giant Branch is still present also in this plot (this metal-rich sequence was discussed in terms of tidal capture of bulge stars by clusters in Bica et al. 1997).

Table 2 lists the coordinates, magnitudes and colours of the sample stars, as obtained from the NTT data. The star's identifications follow our own notation. For each star the first line lists the observed magnitudes, while the second line gives the magnitudes corrected for total extinction. The stars numbers were given in order of magnitude in a selected list of member stars (therefore star 1 is a brighter one in Fig. 2).

\subsection{Spectra}

High resolution spectra of two stars in HP-1, in the wavelength range $\lambda \lambda$ 4800-6800 $\AA$, were obtained with the UVES spectrograph at the ESO VLT. We used essentially only the reddest portion of the spectrum (5800-6800 $\AA$ ) covered by the MIT backside illuminated and AR coated CCD ESO \# 20 of $4096 \times 2048$ pixels, of pixel size $15 \times 15 \mu \mathrm{m}$. With the UVES standard setup 580, the resolution is $R \sim 55000$ for a slit width of $0.8 \mathrm{arcsec}$. The pixel scale is $0.0174 \AA / \mathrm{px}$. The log of observations is shown in Table 3.

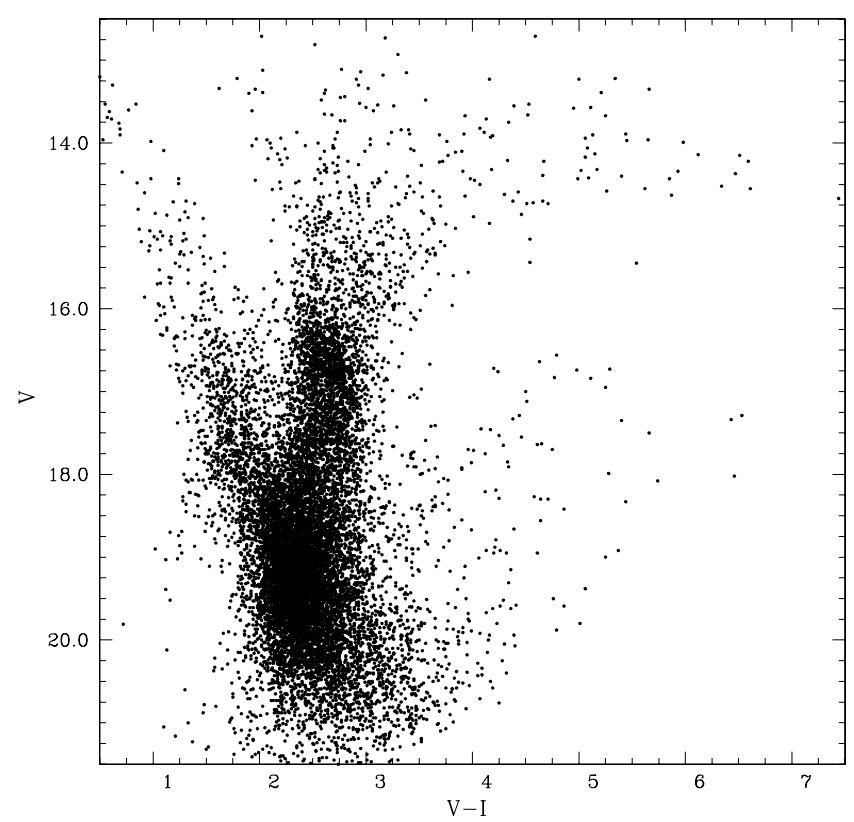

Fig. 3. The WFI $V$ vs. $V-I$ CMD of HP- 1 . Size of extraction is $5^{\prime} \times 5^{\prime}$.

The spectra were reduced using the UVES context of the MIDAS reduction package, including bias and inter-order background subtraction, flatfield correction, extraction and wavelength calibration (Ballester et al. 2000). The two spectra obtained for each star were summed before the analysis. $S / N$ ratio were measured in the co-added spectra, at several wavelength regions encompassing around 2 to $3 \AA$, from 6200 to $6500 \AA$, and the value reported in Table 2 is the mean of these measurements.

The equivalent widths were measured using a new automatic code developed by P. Stetson and E. Pancino (DAOSPEC, Stetson \& Pancino 2006, in preparation), and also measured line by line using IRAF. The agreement is very good between the two methods, but the line by line work was useful to discard lines affected by cosmic rays and blends.)

A radial velocity $v_{\mathrm{r}}=55.7 \pm 0.7 \mathrm{~km} \mathrm{~s}^{-1}$ or heliocentric $v_{\mathrm{r}}^{\text {hel }}=45.8 \mathrm{~km} \mathrm{~s}^{-1}$ was found for HP-1, in good agreement with values of $60 \pm 13 \mathrm{~km} \mathrm{~s}^{-1}$ measured by Minniti (1995) based on 6 stars observed with a $2 \AA$ resolution, and the value of $53.1 \pm 9.8$ reported by Harris (1996, updated in www . physics.mcmaster.ca/Globular.html). The present radial velocity for HP-1 should be preferred over other values, because it is based on high resolution spectra, and expected to be more precise than previous measurements. The radial velocity is consistent with the cluster being a member of the bulge 
Table 3. Log of the spectroscopic observations carried out on 2001 July 5-6 (Julian date 2452 095, 2452 096). The quoted seeing is the mean value along the exposures.

\begin{tabular}{llllllllllc}
\hline \hline Target & $V$ & Date & UT & $\begin{array}{l}\text { Exp. } \\
(\mathrm{s})\end{array}$ & $\begin{array}{l}\text { Seeing } \\
\left({ }^{\prime \prime}\right)\end{array}$ & Airmass & $\begin{array}{l}(S / N) / \mathrm{px} \\
(\mathrm{sum})\end{array}$ & $\begin{array}{c}\text { Slit } \\
\text { width }\end{array}$ & $\begin{array}{c}v_{\mathrm{r}}^{\text {obs }} \\
\mathrm{km} \mathrm{s}^{-1}\end{array}$ & $\begin{array}{c}v_{\mathrm{r}}^{\text {hel }} \\
\mathrm{km} \mathrm{s}^{-1}\end{array}$ \\
\hline 2 & 16.982 & 05.07 .01 & $04: 07: 52.9$ & 5400 & 0.4 & 1.02 & 70 & $0.8^{\prime \prime}$ & $+54.62 \pm 0.85$ & 44.81 \\
2 & & 05.07 .01 & $05: 38: 46.5$ & 5400 & 0.4 & 1.17 & & $0.8^{\prime \prime}$ & $+54.31 \pm 0.77$ & 44.36 \\
3 & 17.003 & 05.07 .01 & $07: 30: 50.5$ & 3500 & 0.8 & 1.75 & 45 & $0.8^{\prime \prime}$ & $+56.73 \pm 0.83$ & 46.64 \\
3 & & 06.07 .01 & $23: 05: 47.0$ & 5400 & 0.5 & 1.76 & & $0.8^{\prime \prime}$ & $+57.18 \pm 0.60$ & 47.41 \\
\hline
\end{tabular}

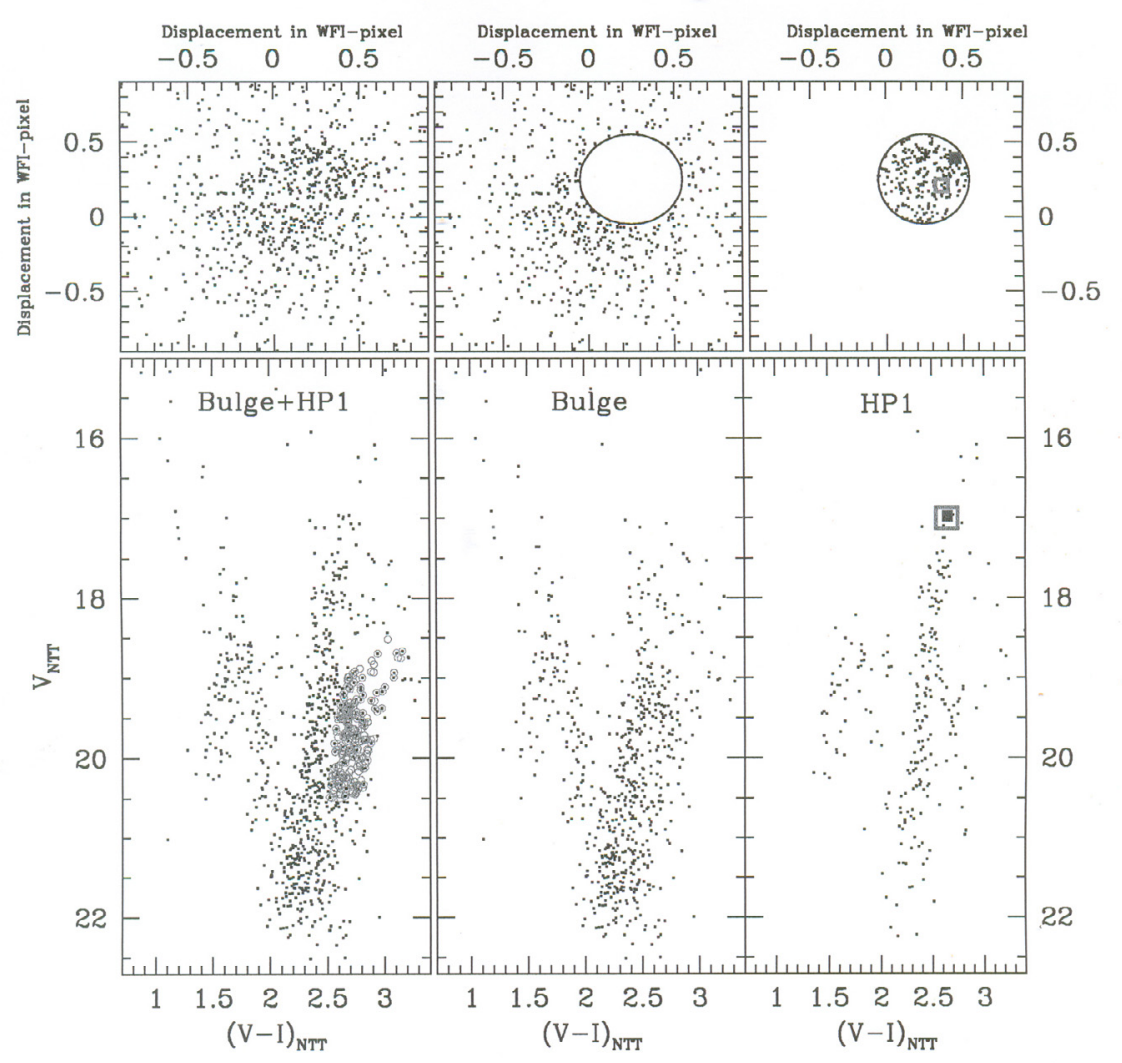

Fig. 4. The WFI and NTT data used to establish proper motion cleaning of the field.

population, rather than being a halo cluster near perigalacticon. The determination of the proper motion would nevertheless be necessary to rule out completely this latter alternative.

\section{Proper motion and membership confirmation}

In an attempt to ascertain the membership of our 2 stars, we make use of the two photometric catalogs (based on NTT and WFI data) and search for a possible signature of the HP-1 proper motion.

We emphasize that our approach is only tentative, since we do not correct for geometrical distorsion of the two catalogs obtained at two different telescopes. Moreover, a bias due to the different photometric filters is expected. Keeping all this in mind, we simply fix the coordinates in the WFI catalog and estimate the displacement of the NTT data, that are separated by 8 years. Following Bedin et al. (2001) we show the proper motion and colour-magnitude diagram of this comparison.
The lower left panel of Fig. 4 shows the colour-magnitude diagram of stars matched in the WFI and NTT catalogs. Plotted as grey open circles are the reference stars with respect to which we estimate the displacement. These are clearly bulge red giants. The upper left panel shows the displacement of the stars, calculated in WFI pixels. Since the reference stars were taken to be bulge members, their mean proper motion is expected to be zero. Although there is a visible dispersion, we note a concentration of stars [centered at $(\mathrm{d} x, \mathrm{~d} y)=$ $(+0.25,+0.25)]$, and offset from the zero mean proper motion.

The middle panels show the proper motion and colourmagnitude diagram of stars selected outside an arbitrary 0.3 pixel radius from $(\mathrm{d} x, \mathrm{~d} y)=(+0.25,+0.25)$, whereas the right panels show the diagrams for stars selected within this radius. Comparing the two colour-magnitude diagrams, we note a hint of the separation of cluster members from bulge stars. Indeed, the middle panel shows a typical " $V$ "-shape bulge diagram composed of young (blue) main sequence stars and their 
Table 4. Photometric stellar parameters derived using the calibrations by Alonso et al. (1999) = AAM99 for $V-I, V-K, J-K$, bolometric corrections, bolometric magnitudes and corresponding gravity $\log g$, and spectroscopic parameters.

\begin{tabular}{|c|c|c|c|c|c|c|c|c|c|c|c|c|c|}
\hline \multirow[b]{2}{*}{ star } & \multicolumn{2}{|r|}{$\overline{T_{\text {eff }}}$} & \multicolumn{3}{|c|}{ AAM99 } & \multirow[b]{2}{*}{$\mathrm{BC}_{\mathrm{V}}$} & \multirow[b]{2}{*}{$M_{\mathrm{bol}}$} & \multirow[b]{2}{*}{$\log g$} & \multicolumn{4}{|c|}{ Spectroscopic } & parameters \\
\hline & $V-I$ & $V-K$ & $J-H_{\mathrm{TCS}}$ & $J-K_{\mathrm{TCS}}$ & mean & & & & $T_{\text {eff }}$ & $\log g$ & {$[\mathrm{FeI} / \mathrm{H}][$} & $\mathrm{eII} / \mathrm{H}][\mathrm{Fe} / \mathrm{H}] v$ & $v_{\mathrm{t}}\left(\mathrm{km} \mathrm{s}^{-1}\right)$ \\
\hline HP1-2 & 4418 & 4312 & 4518 & 4592 & 4466 & -0.53 & -0.69 & 1.70 & 4630 & 1.7 & -1.02 & $\begin{array}{ll}-0.97 & -1.0\end{array}$ & 1.60 \\
\hline HP1-3 & 4426 & 4212 & 4268 & 4326 & 4314 & -0.60 & -0.61 & 1.69 & 4450 & 1.75 & -0.99 & $-0.95-0.97$ & 1.40 \\
\hline
\end{tabular}

respective red giants. We note however that some horizontal branch stars (most probably HP-1 members) remain in this diagram. On the other hand, the right panel shows a colourmagnitude diagram where one can easily identify a cleaned HP-1 red giant and horizontal branch stars. Thus, although the separation between the two populations is not perfect, we can argue to have separated the bulk motion of HP-1.

Keeping in mind all the uncertainties and the large dispersion of bulge proper motion, we note that the 2 stars of interest show a proper motion that respects that of mean cluster motion.

\section{Stellar parameters}

\subsection{Temperatures}

In order to obtain a first guess temperature and gravity from the star colours, it is necessary to correct for insterstellar extinction. Armandroff \& Zinn (1988) derived $E(B-V)=1.44$ based on the interstellar band at $8621 \AA$. Minniti (1995) derived $E(J-K)=0.94$, and $E(B-V)=1.68$ using $E(J-K)=$ $0.56 E(B-V)$. A reddening of $E(V-I)=1.58$ converting to $E(B-V)=1.19$, and a distance modulus $(m-M)_{0}=14.15$ were derived by Ortolani et al. (1997), from a comparison with the CMD of NGC 6752. Harris (1996) gives $E(B-V)=0.74$ and $(m-M)_{V}=18.03$ for HP-1, significantly different from both Minniti (1995) and Ortolani et al. (1997), despite this latter paper being quoted as source for the reddening.

We initially adopted $E(B-V)=1.19$ from Ortolani et al. (1997). However this reddening value had been derived from a CMD comparison with the cluster NGC 6752, of metallicity $[\mathrm{Fe} / \mathrm{H}]=-1.54$ (Harris 1996). With this reddening we derived a metallicity of $[\mathrm{Fe} / \mathrm{H}]=-1.05$ for $\mathrm{HP}-1$. In an iterative procedure, we then re-derived the reddening using the mean locus of the higher metallicity clusters M 5 (NGC 5904), NGC 6171 and 47 Tuc, with $[\mathrm{Fe} / \mathrm{H}]=-1.27 /-1.38 /-1.12,-1.04 /-1.09 /-0.95$, $-0.76 /-0.71 /-0.78$ according to Harris (1996), Zinn \& West (1984) and Carretta \& Gratton (1997). Reddening values of $E(B-V)=1.11,1.12$ and 1.15 are found relative to these three clusters. We adopted $E(B-V)=1.12$, corresponding to the average of reddening values derived by comparison with the clusters M 5, NGC 6171 and 47 Tuc.

For the present analysis we adopt the extinction law given by Dean et al. (1978) and Rieke \& Lebofsky (1985), namely:

$$
\begin{aligned}
& R_{V}=A_{V} / E(B-V)=3.1 \\
& E(V-I) / E(B-V)=1.33 \\
& E(V-K) / E(B-V)=2.744 \\
& E(J-K) / E(B-V)=0.527
\end{aligned}
$$

implying:

$$
\begin{aligned}
& A_{I} / E(B-V)=1.77 ; \quad A_{J} / E(B-V)=0.88 \\
& A_{H} / E(B-V)=0.55 ; \quad A_{K} / E(B-V)=0.356
\end{aligned}
$$

Effective temperatures were derived from $V-K, V-I$ and $J-K$ using the colour-temperature calibrations of Alonso et al. $\left(1999\right.$, hereafter AAM99), and using $(V-I)_{C}=0.778(V-I)_{J}$ (Bessell 1979). The $J, H, K_{S}$ magnitudes and colours were transformed from the 2MASS system to CIT (California Institute of Technology), and from this to TCS (Telescopio Carlos Sánchez), using the relations established by Carpenter (2001) and Alonso et al. (1998). The derived effective temperatures are listed in Table 4 . Given that the $V-K$ colour is more sensitive to effective temperature, we adopted a compromise value between $T_{(V-K)}$ and the mean given in Table 4, namely $T_{\text {eff }}=4450 \mathrm{~K}$ for HP1-2 and $4300 \mathrm{~K}$ for HP1-3 as first guess.

\subsection{Gravities}

The classical relation:

$\log g_{*}=4.44+4 \log \frac{T_{*}}{T_{\odot}}+0.4\left(M_{\mathrm{bol}}-4.75\right)+\log \frac{M_{*}}{M_{\odot}}$

was used, adopting $T_{\odot}=5770 \mathrm{~K}, M_{*}=0.85 M_{\odot}$ and $M_{\text {bol } \odot}=$ 4.75 (Cram 1999).

A distance modulus of $(m-M)_{0}=14.15$ was adopted from Ortolani et al. (1997) together with a total extinction $A_{V}=3.47$, as discussed in Sect. 4.1. The bolometric corrections from AAM99 and corresponding gravities are given in Table 4.

\section{Atomic and molecular data}

The Fe I line list and respective oscillator strengths given in NIST (Martin et al. 2002) were used to derive spectroscopic parameters. Six measurable Fe II lines, and their respective oscillator strengths from Biémont et al. (1991), and renormalized by Meléndez \& Barbuy (2006, in preparation), were used to check whether ionization equilibrium was verified.

In Zoccali et al. (2004) the $g f$ values of NaI, MgI, SiI, CaI, TiI and TiII lines were adopted from values selected in the literature, from a comparison to the solar spectrum observed with the same VLT-UVES instrumentation ${ }^{1}$ as the sample stars. NMARCS solar atmospheric model (Edvardsson et al. 1993) was used in order to be compatible with the model atmosphere grid used for the target giant stars. These values are

\footnotetext{
1 Available from: www.eso.org/observing/dfo/quality/ UVES/pipeline/solar_spectrum.html
} 
further revised in the present paper, using the same solar spectrum and model atmosphere, with the following procedure: in cases where the fit to the Sun could be further improved relative to the literature $g f$ value, we changed them until a best fit was obtained. The calculation was then applied to Arcturus, using the spectrum of Hinkle et al. (2000), and adopting the stellar parameters derived by Meléndez et al. (2003), namely $T_{\text {eff }}=4275 \mathrm{~K}, \log g=1.55,[\mathrm{Fe} / \mathrm{H}]=-0.54, v_{\mathrm{t}}=$ $1.65 \mathrm{~km} \mathrm{~s}^{-1}$ and abundances $[\mathrm{C} / \mathrm{Fe}]=-0.08,[\mathrm{~N} / \mathrm{Fe}]=+0.3$, $[\mathrm{O} / \mathrm{Fe}]=+0.43$. Abundance ratios in Arcturus were rederived for $[\mathrm{Na} / \mathrm{Fe}],[\mathrm{Mg} / \mathrm{Fe}],[\mathrm{Al} / \mathrm{Fe}],[\mathrm{Si} / \mathrm{Fe}],[\mathrm{Ca} / \mathrm{Fe}]$, and $[\mathrm{Ti} / \mathrm{Fe}]$ as given in Table 6.

The damping constants were computed where possible, and in particular for most of the Fe I lines, using the collisional broadening theory of Barklem et al. (1998, 2000, and references therein). The use of quantum mechanical computations of $C_{6}$ applied to $\mathrm{Ca}$ lines are discussed for example in Cayrel et al. (1996), where however, it was concluded that the good match of the computed $C_{6}$ to observed spectra is model dependent - see also Barbuy et al. (2003). Coelho et al. (2005, see discussion in the Appendix) found that, using the solar ATLAS9 model, $5 \%$ of the lines for which the broadening values were calculated through the Barklem et al. theory have too strong wings when compared with the observed spectrum. We adopted a mean of $\gamma($ Barklem $) / \gamma($ best fit $) \approx 1.5$. Note that $\gamma$ values are compared and not $C_{6}$ values. The correction factor in $C_{6}$ is variable, and essentially goes up to a factor 3 (Barbuy et al. 2003; Coelho et al. 2005). Whereas Coelho et al. (2005) applied a factor $1 / 3$ decrease to all $\mathrm{Ca}$ lines, we adopt here a more conservative decrease of $2 / 3$ to all $\mathrm{Ca}$ and $\mathrm{Ti}$ lines. Given this fact, combined with the fact that we found very low $\mathrm{Ca}$ abundances in Zoccali et al. (2004) and Alves-Brito et al. (2005), we decided to reverify our atomic parameters. We redetermined $\log g f$ values of $C$ a lines, using now the $2 / 3 C_{6}$ (Barklem et al.). The newly adopted $C_{6}$ and $\log g f$ values for $C$ a lines are given in Table 6 (for the $6102.727 \AA$ line the Barklem et al. $C_{6}$ original value was kept, since it appeared to give a better fit).

For the forbidden oxygen line [OI]6300 $\AA$ we adopt the oscillator strength recently derived by Allende Prieto et al. (2001): $\log g f=-9.716$.

For lines of the heavy elements BaII, LaII and EuII, a hyperfine structure was taken into account, based on the hyperfine constants by Lawler et al. (2001a) for EuII, Lawler et al. (2001b) for LaII and Biehl (1976) for BaII.

Molecular lines of $\mathrm{CN}\left(\mathrm{A}^{2} \Pi-\mathrm{X}^{2} \Sigma\right), \mathrm{C}_{2}$ Swan $\left(\mathrm{A}^{3} \Pi-\mathrm{X}^{3} \Pi\right)$, $\mathrm{TiO}\left(\mathrm{A}^{3} \Phi-\mathrm{X}^{3} \Delta\right) \gamma$ and $\mathrm{TiO}\left(\mathrm{B}^{3} \Pi-\mathrm{X}^{3} \Delta\right) \gamma^{\prime}$ systems are taken into account.

Solar abundances, reported in Table 7, were adopted from Grevesse \& Sauval (1998), except for the value for oxygen where $\epsilon(\mathrm{O})=8.77$ was assumed, as recommended by Allende Prieto et al. (2001) for the use of 1D model atmospheres.

\section{Iron abundances}

Photospheric 1D models for the sample giants were extracted from the NMARCS grid (Plez et al. 1992), originally developed by Bell et al. (1976) and Gustafsson et al. (1975).
The LTE abundance analysis and the spectrum synthesis calculations were performed using the codes by Spite (1967), with the introduction of molecular bands by Barbuy (1982), and described in Cayrel et al. (1991), Barbuy et al. (2003) and Coelho et al. (2005). An Iron abundance of $\epsilon(\mathrm{Fe})=7.50$ (Grevesse \& Sauval 1998) was adopted, as reported in Table 7.

The line list of FeI and FeII lines was selected by erasing lines with equivalent widths $E W>130 \mathrm{~m} \AA$ and excitation potential $\chi_{\text {exc }}<2.0$. This avoids the stronger lines, and resonance lines for which NLTE effects are more important (Asplund 2005; Thévenin \& Idiart 1999). The line list of Fe lines, together with measured equivalent widths is given in Table A.1.

The stellar parameters were derived by initially adopting the photometric effective temperature and gravity, and then further constraining the temperature by imposing excitation equilibrium for Fe I lines. A few Fe II lines were reliable, allowing to derive gravities imposing agreement between Fe I and Fe II abundances (ionization equilibrium). Microturbulence velocities $v_{\mathrm{t}}$ were determined by canceling the trend of Fe I abundance vs. equivalent width.

The spectroscopic parameters $T_{\text {eff }}, \log g,[\mathrm{Fe} \mathrm{I} / \mathrm{H}],[\mathrm{Fe} \mathrm{II} / \mathrm{H}]$, $[\mathrm{Fe} / \mathrm{H}]$ and $v_{\mathrm{t}}$ values are reported in the last columns of Table 4 and they were adopted for the derivation of abundance ratios.

\subsection{Photometric vs. spectroscopic parameters}

Adopting the photometric parameters given in Table 4 at face value, namely $\left(T_{\mathrm{eff}}, \log g\right)=(4450,1.7)$ and $(4300,1.7)$ for HP1-1 and HP1-2 respectively, the excitation and ionization equilibria are not fulfilled. It is interesting in any case to mention that, adopting these parameters, a metallicity of $[\mathrm{Fe} / \mathrm{H}]=-1.25$ is obtained for a microturbulent velocity of $1.5 \mathrm{~km} \mathrm{~s}^{-1}$.

\subsection{Errors}

In this section we estimate the errors valid within the spectroscopic parameter determination. The error on the slope in the FeI vs. ionization potential implies an error in the temperature of $\pm 100 \mathrm{~K}$ for the two sample stars. In Table 5 are reported the errors for the star HP1-3, on the FeI and other element abundance ratios, induced by a change of $\Delta T_{\text {eff }}=+100 \mathrm{~K}$, $\Delta \log g=+0.3, \Delta v_{\mathrm{t}}$ of $0.2 \mathrm{~km} \mathrm{~s}^{-1}$ (the rms of the data points of excitation equilibrium shows an uncertainty of the order of $0.2 \mathrm{~km} \mathrm{~s}^{-1}$ on the microturbulence velocity). The total error is given in the last column.

\section{Abundance ratios}

Abundances ratios were obtained through line-by-line spectrum synthesis calculations compared to the observed lines, for the line list given in Tables 6 , where the $g f$-values and damping constants used are also reported.

The fit for the Sun, Arcturus and HP1-2 are shown in Figs. 5-10 for a few CaI, SiI and TiI lines. Figures 11 and 12 show the fits to the MgI triplet at 6318 and the Eu $6645 \AA$ line, respectively. 
Table 5. Abundance uncertainties for a $\Delta T_{\text {eff }}=100 \mathrm{~K}, \Delta \log g=+0.3$, $\Delta v_{\mathrm{t}}=0.2 \mathrm{~km} \mathrm{~s}^{-1}$ and corresponding total error. The errors mean the difference in abundance that needs to be added in order to fit the observed spectrum. The values given are a mean derived from the errors from each line.

\begin{tabular}{lcccc}
\hline \hline Abundance & $\begin{array}{c}\Delta T \\
(100 \mathrm{~K})\end{array}$ & $\begin{array}{c}\Delta \log g \\
(+0.3 \mathrm{dex})\end{array}$ & $\begin{array}{c}\Delta v_{\mathrm{t}} \\
\left(+0.2 \mathrm{kms}^{-1}\right)\end{array}$ & $\left(\sum x^{2}\right)^{1 / 2}$ \\
$(1)$ & $(2)$ & $(3)$ & $(4)$ & $(5)$ \\
\hline \multicolumn{5}{c}{$\mathrm{HP} 1-3$} \\
\hline $\mathrm{FeI} / \mathrm{H}]$ & -0.07 & -0.07 & +0.07 & 0.12 \\
{$[\mathrm{O} / \mathrm{Fe}]$} & +0.00 & +0.15 & 0.00 & 0.15 \\
{$[\mathrm{NaI} / \mathrm{Fe}]$} & +0.03 & +0.05 & 0.00 & 0.05 \\
{$[\mathrm{MgI} / \mathrm{Fe}]$} & +0.01 & +0.05 & 0.00 & 0.06 \\
{$[\mathrm{SiI} / \mathrm{Fe}]$} & -0.09 & +0.10 & -0.02 & 0.14 \\
{$[\mathrm{CaI} / \mathrm{Fe}]$} & +0.10 & +0.10 & -0.03 & 0.15 \\
{$[\mathrm{TiI} / \mathrm{Fe}]$} & +0.12 & +0.04 & -0.01 & 0.13 \\
{$[\mathrm{TiII} / \mathrm{Fe}]$} & -0.01 & +0.15 & -0.01 & 0.15 \\
{$[\mathrm{BaII} / \mathrm{Fe}]$} & +0.02 & +0.05 & -0.15 & 0.16 \\
{$[\mathrm{LaII} / \mathrm{Fe}]$} & 0.00 & +0.12 & 0.00 & 0.12 \\
{$[\mathrm{EuII} / \mathrm{Fe}]$} & +0.02 & +0.15 & 0.00 & 0.15 \\
\hline
\end{tabular}

Table 7. Final abundance ratios $[\mathrm{X} / \mathrm{Fe}]$ of the program stars, compared to those of the metal-poor bulge clusters UKS 1 (Origlia et al. 2005) and Terzan 4 (Origlia et al. 2004). In the 2nd column the solar abundances adopted are reported. No. corresponds to the number of lines used.

\begin{tabular}{lcrrrrrr}
\hline \hline Species & $\epsilon(\mathrm{X})_{\odot}$ & No. & HP1-2 & HP1-3 & Mean & UKS 1 & Terzan 4 \\
\hline$[\mathrm{Fe} / \mathrm{H}]$ & 7.50 & 50 & -1.00 & -0.97 & -0.99 & -0.78 & -1.60 \\
{$[\mathrm{OI} / \mathrm{Fe}]$} & 8.77 & 1 & +0.40 & +0.40 & +0.40 & +0.27 & +0.55 \\
{$[\mathrm{NaI} / \mathrm{Fe}]$} & 6.33 & 2 & +0.00 & +0.00 & +0.00 & - & - \\
{$[\mathrm{MgI} / \mathrm{Fe}]$} & 7.58 & 4 & +0.00 & +0.20 & +0.10 & +0.32 & +0.41 \\
{$[\mathrm{SiI} / \mathrm{Fe}]$} & 7.55 & 8 & +0.30 & +0.30 & +0.30 & +0.28 & +0.55 \\
{$[\mathrm{CaI} / \mathrm{Fe}]$} & 6.36 & 16 & -0.04 & +0.10 & +0.03 & +0.38 & +0.53 \\
{$[\mathrm{TiI} / \mathrm{Fe}]$} & 5.02 & 17 & +0.04 & +0.00 & +0.02 & +0.32 & +0.43 \\
{$[\mathrm{TiII} / \mathrm{Fe}]$} & & 3 & +0.10 & +0.15 & +0.13 & - & \\
{$[\mathrm{BaII} / \mathrm{Fe}]$} & 2.13 & 2 & +0.10 & +0.20 & +0.15 & - & \\
{$[\mathrm{LaII} / \mathrm{Fe}]$} & 1.22 & 1 & 0.00 & 0.00 & +0.00 & - & \\
{$[\mathrm{EuII} / \mathrm{Fe}]$} & 0.51 & 1 & +0.00 & +0.30 & +0.15 & - & \\
\hline
\end{tabular}

The odd-Z element sodium, built up during carbon burning, shows a solar ratio: $[\mathrm{Na} / \mathrm{Fe}] \sim 0.0$. The $\alpha$-elements $\mathrm{O}$ and $\mathrm{Si}$ are enhanced by: $[\mathrm{O} / \mathrm{Fe}]=+0.4,[\mathrm{Si} / \mathrm{Fe}]=+0.3$, whereas $\mathrm{Mg}$, shows a lower enhancement of $[\mathrm{Mg} / \mathrm{Fe}]=+0.1$, and $\mathrm{Ca}$ and $\mathrm{Ti}$ show solar values $[\mathrm{Ca} / \mathrm{Fe}]=[\mathrm{Ti} / \mathrm{Fe}]=+0.0$. We believe that oxygen and silicon lines are more reliable, than those of the other $\alpha \mathrm{s}$. The reason for saying this is that $\mathrm{Mg}$ lines (Fig. 11) are weak, and from their behaviour in different stars, there is some evidence that there might be some unidentified lines blending the $\mathrm{Mg}$ ones. Among the $\mathrm{Ca}$ and $\mathrm{Ti}$ lines, several of them are strong, where both $C_{6}$ and $\log g f$ have to be fitted, and they are therefore subject to uncertainties due to the interdependence between $C_{6}$ and $\log g f$; besides, some of them are blended. On the other hand, for these elements a rather large number of lines are available, which lead to more

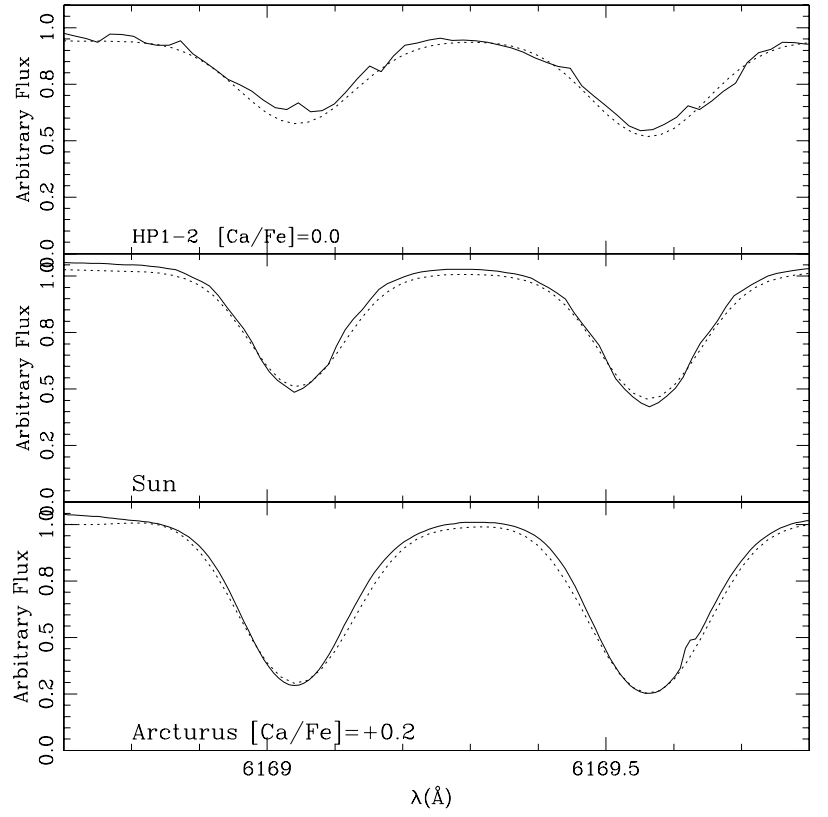

Fig. 5. CaI 6169.044 and $6169.564 \AA$ lines shown for the solar, Arcturus, and HP1-2 spectra.

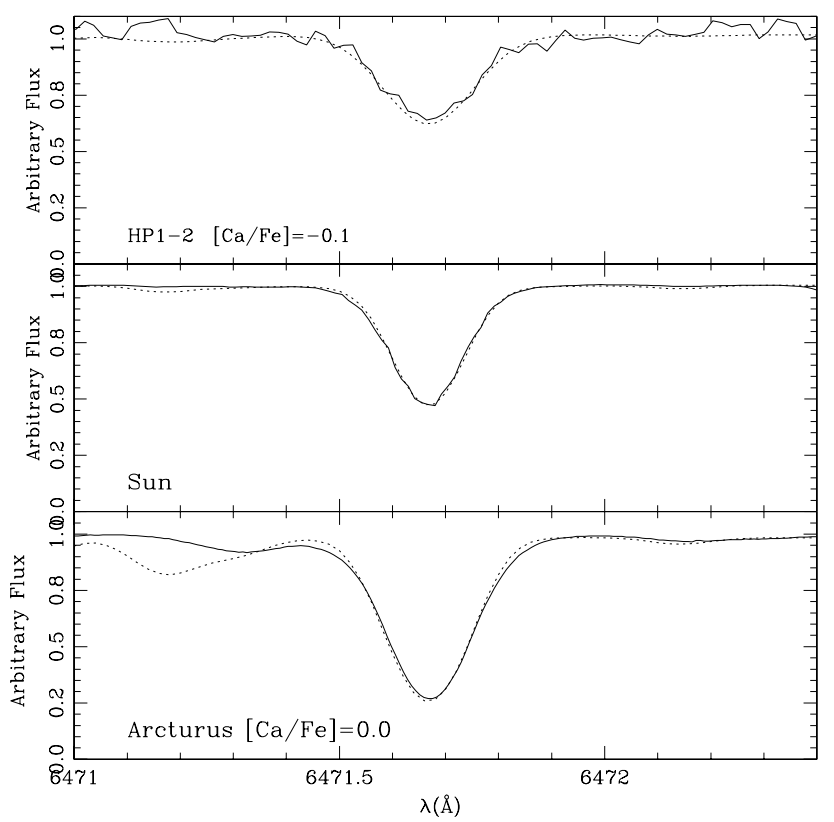

Fig. 6. CaI $6471.668 \AA$ Alne shown for the solar, Arcturus, and HP1-2 spectra.

reliable results. The r-process element $\mathrm{Eu}$ is somewhat enhanced: $[\mathrm{Eu} / \mathrm{Fe}]=+0.15$ whereas the s-element La shows a solar ratio $[\mathrm{La} / \mathrm{Fe}]=0.0$ and $\mathrm{Ba}$ is enhanced with $[\mathrm{Ba} / \mathrm{Fe}]=+0.2$.

\section{Discussion and conclusions}

We have derived element abundances for two giants in the globular cluster HP-1. A metallicity of $[\mathrm{Fe} / \mathrm{H}] \sim-1.0 \pm 0.2$ is found. It is considerably higher than that derived from the RGB slope and HB morphology by Ortolani et al. (1997). We have confirmed through proper motion analysis that the two stars are cluster members. Therefore HP-1 is unusual in possessing 


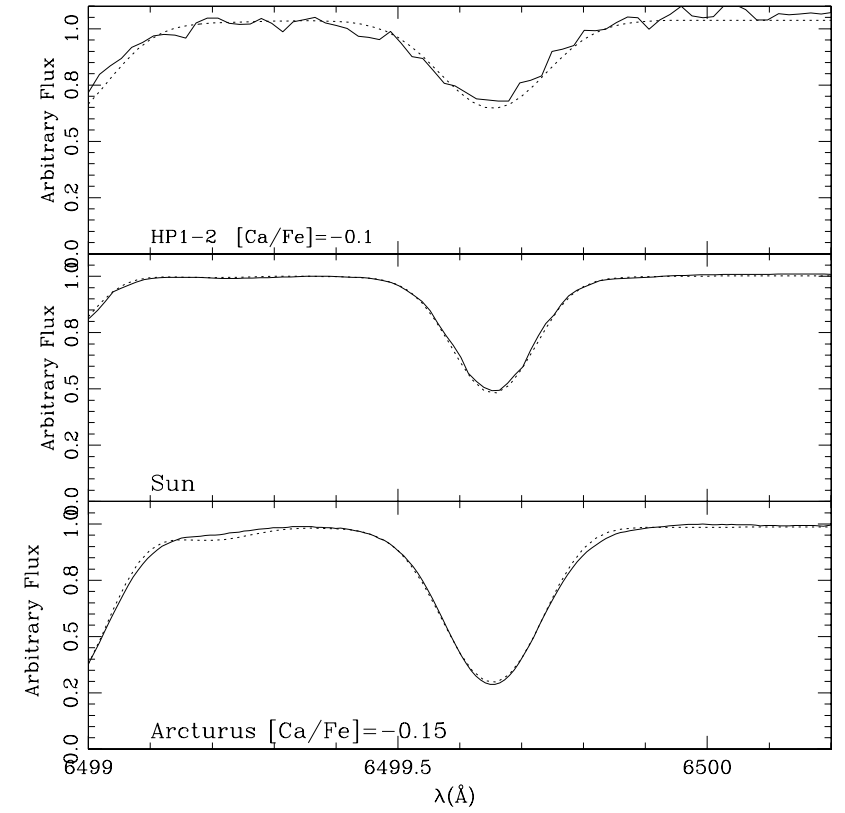

Fig. 7. CaI $6499.654 \AA$ A line shown for the solar, Arcturus, and HP1-2 spectra.

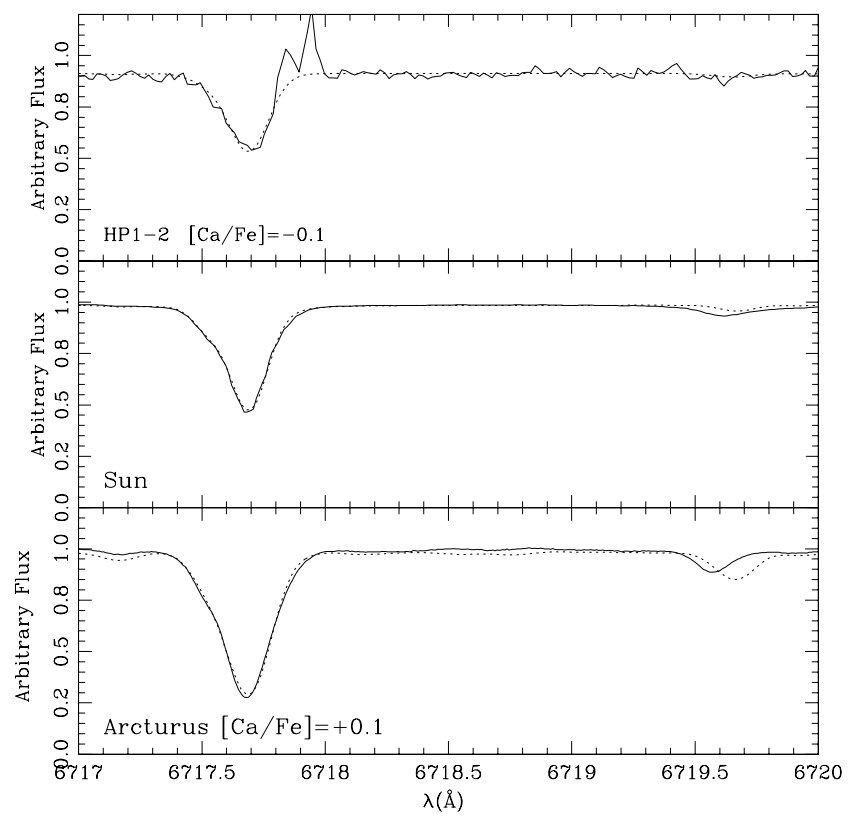

Fig. 8. CaI $6717.687 \AA$ line shown for the solar, Arcturus, and HP1-2 spectra.

both a blue Horizontal Branch and a relatively high metallicity. This cluster could be considered similar to NGC 6388 and NGC 6441 (Rich et al. 1997), both bulge members, known to have blue HBs and high metallicity $[\mathrm{Fe} / \mathrm{H}] \sim-0.6$. HP-1 differs from these two clusters in the sense that it has a BHB like a normal metal-poor cluster, whereas NGC 6388 and NGC 6441 have both a blue and a populous red HB. It is more difficult to reconcile our iron abundance with the steep slope of the Red Giant Branch; the slope of HP 1 is consistent with $[\mathrm{Fe} / \mathrm{H}] \approx-1.5$ like NGC 6752 . The RGB slope is reflection of line blanketing in the bluer bands. We find HP-1 to be enhanced in the $\alpha$ elements $\mathrm{O}$ and $\mathrm{Si}$, but Ti is Solar. We speculate

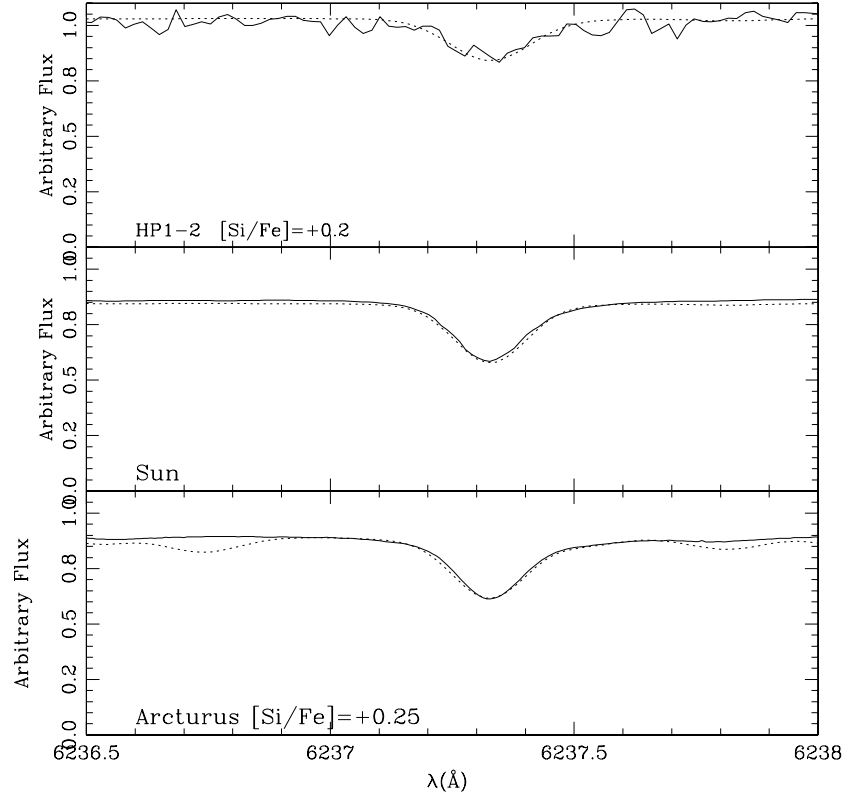

Fig. 9. SiI $6237.328 \AA$ line shown for the solar, Arcturus, and HP1-2 spectra.

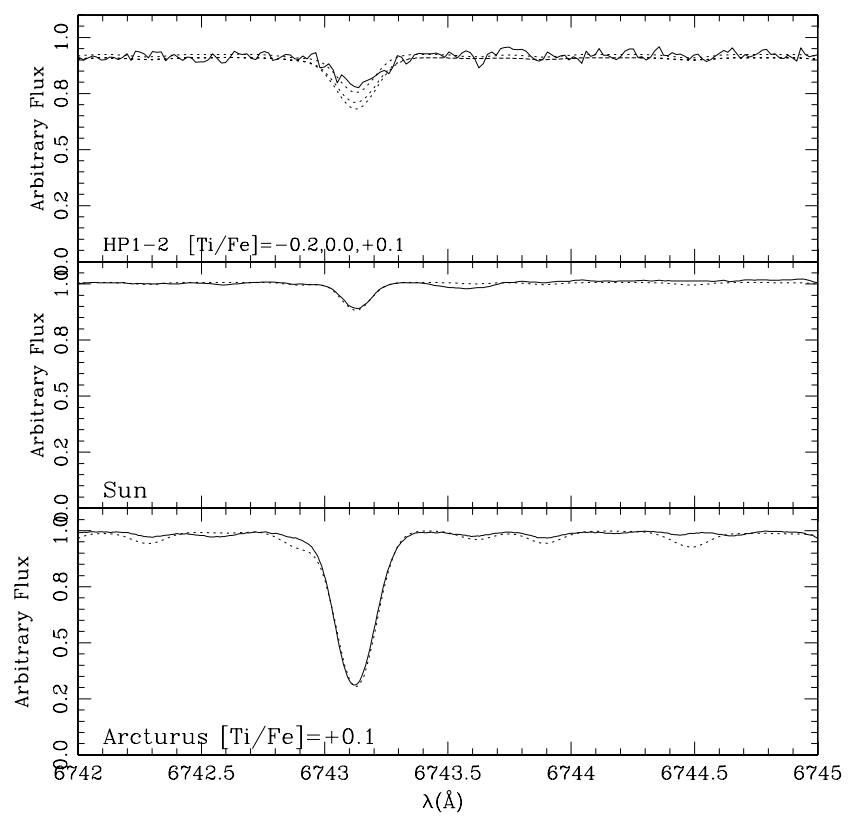

Fig. 10. TiII $6491.58 \AA$ Aine shown for the solar, Arcturus, and HP1-2 spectra.

that the TiO blanketing in HP-1 might be lower than in other clusters of similar metallicity. It would be interesting to explore whether these features are characteristic of other metal poor blue HB globular clusters in the bulge.

It is also possible that field stars have masqueraded as cluster members in both the proper motion and colour-magnitude space; additional stars in this cluster should be the subject of abundance analysis.

Chemical evolution model predictions by Matteucci et al. (1999) indicate that enhancements of $\mathrm{O}, \mathrm{Si}, \mathrm{Mg}, \mathrm{Ca}$ and $\mathrm{Na}$ by amounts of $[\alpha / \mathrm{Fe}] \sim 0.8$ are expected. Our results show enhancements of $[\mathrm{O} / \mathrm{Fe}]=+0.4$ and $[\mathrm{Si} / \mathrm{Fe}]=+0.3$, 


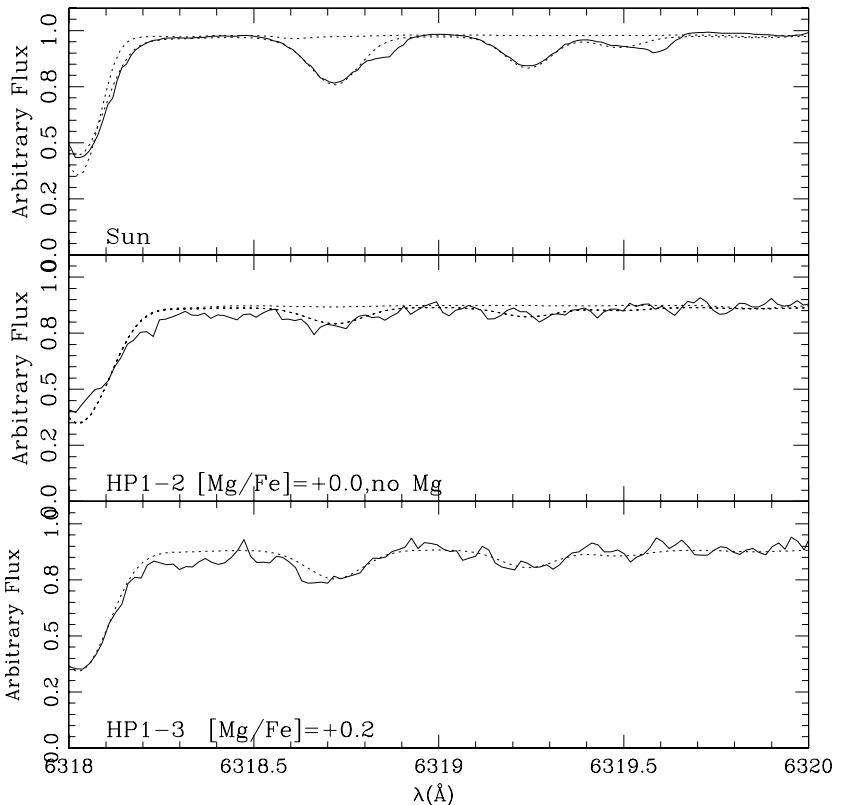

Fig. 11. MgI 6318.72, 6319.24, $6319.45 \AA$ lines shown for the solar, HP1-2 and HP1-3 spectra.

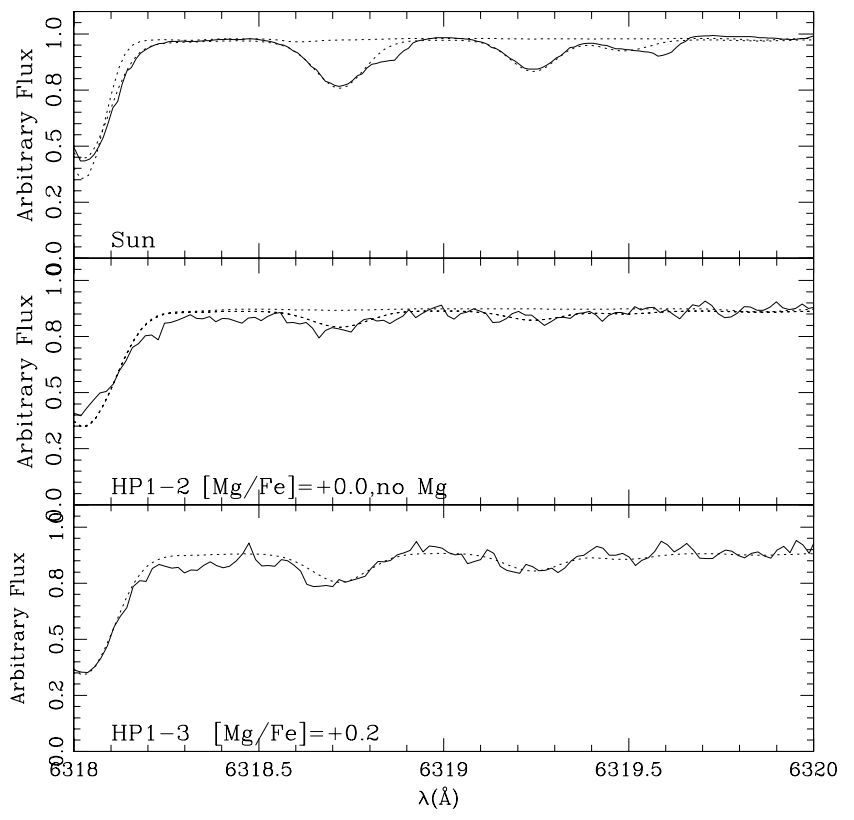

Fig. 12. EuII $6645.127 \AA$ line shown for the Arcturus, HP1-2 and HP1-3 spectra.

$[\mathrm{Mg} / \mathrm{Fe}]=+0.1$ and $[\mathrm{Ca} / \mathrm{Fe}]=[\mathrm{Ti} / \mathrm{Fe}]=+0.0$. Differences in the enhancements of different $\alpha$-elements were also found for example by McWilliam \& Rich $(1994,2003)$ and Pompéia et al. (2003). No enhancements are found for the odd-Z element $\mathrm{Na}[\mathrm{Na} / \mathrm{Fe}] \sim 0.0$. All enhancements are lower than predicted by Matteucci et al. (1999). Still, oxygen and silicon enhancements, together with that of the r-process element $\mathrm{Eu}[\mathrm{Eu} / \mathrm{Fe}]=+0.15$ may be indicative of a fast early enrichment by Supernovae type II. Also, the present $\alpha$-enhancements are compatible with results for the bulge field stars found by McWilliam \& Rich (2003). On the other hand, the solar ratios of $\mathrm{Mg}, \mathrm{Ca}$ and $\mathrm{Ti}$ may be indicative of important contribution by SN Ia type. Therefore in terms of formation of globulars in the bulge, the results are not conclusive.

The s-element La shows a solar ratio $[\mathrm{La} / \mathrm{Fe}]=0.0$ and $\mathrm{Ba}$ is enhanced with $[\mathrm{Ba} / \mathrm{Fe}]=+0.2$. An enhanced Barium abundance is also found in relatively metal-rich globular clusters such as 47 Tucanae (Alves-Brito et al. 2005) M 71 (Ramírez \& Cohen 2002) and M 4 (Ivans et al. 1999).

Finally, a comparison with the results by Origlia \& Rich (2004) for the metal-poor cluster Terzan 4, also located in the inner bulge, of $[\mathrm{Fe} / \mathrm{H}]=-1.6$, for which significant enhancements of $\alpha$-elements were found, may indicate a halo origin, whereas HP-1 showing a more peculiar pattern might be revealing characteristics of the bulge chemical enrichment. It would be of great interest to have further analyses of individual stars in HP-1 and other metal-poor bulge globular clusters.

Acknowledgements. B.B. and E.B. acknowledge grants from $\mathrm{CNPq}$ and Fapesp. D.M. acknowledges grants from FONDAP Center for Astrophysics 15010003. DM also thanks the support from the John Simon Guggenheim Foundation. S.O. acknowledges the Italian Ministero dell'Università e della Ricerca Scientifica e Tecnologica (MURST) under the program on "Fasi iniziali di Evoluzione dell'Alone e del Bulge Galattico" (Italy). This publication makes use of data products from the Two Micron All Sky Survey, which is a joint project of the University of Massachusetts and the Infrared Processing and Analysis Center/California Institute of Technology, funded by the National Aeronautics and Space Administration and the National Science Foundation.

\section{References}

Allende Prieto, C., Lambert, D. L., \& Asplund, M. 2001, ApJ, 556, L63

Alonso, A., Arribas, S., \& Martínez-Roger, C. 1998, A\&AS, 131, 209

Alonso, A., Arribas, S., \& Martínez-Roger, C. 1999, A\&AS, 140, 261 (AAM99)

Alves-Brito, A., Barbuy, B., Ortolani, S., et al. 2005, A\&A, 435, 657

Armandroff, T. E., \& Zinn, R. 1988, AJ, 96, 92

Asplund, M. 2005, ARA\&A, 43, 481

Ballester, P., Modigliani, A., Boitquin, O., et al. 2000, The Messenger, 101,31

Barbuy, B. 1982, Ph.D. Thesis, Université de Paris VII

Barbuy, B., Bica, E., \& Ortolani, S. 1998, A\&A, 333, 117

Barbuy, B., Perrin, M.-N., Katz, D., et al. 2003, A\&A, 404, 661

Barklem, P. S., Anstee, S. D., \& O'Mara, B. J. 1998, PASA, 15, 336

Barklem, P. S., Piskunov, N. E., \& O'Mara, B. J. 2000, A\&AS, 142, 467

Bedin, L. R., Anderson, J., King, I. R., \& Piotto, G. 2001, ApJ, 560, L75

Bell, R. A., Eriksson, K., Gustafsson, B., \& Nordlund, A. 1976, A\&AS, 23, 37

Bensby, T., Feltzing, S., \& Lundström, I. 2003, A\&A, 410, 527

Bessell, M. S. 1979, PASP, 91, 589

Bica, E., Dottori, H., Rodrigues de Oliveira Filho, I., Ortolani, S., \& Barbuy, B. 1997, ApJ, 482, L49

Bica, E., Bonatto, C., Barbuy, B., \& Ortolani, S. 2005, A\&A, in press Biehl, D., 1976, Ph.D. Thesis, University of Kiel

Biémont, E., Baudoux, M., Kurucz, R. L., Ansbacher, W., \& Pinnington, A. E. 1991, A\&A, 240, 539

Carpenter, J. M. 2001, AJ, 121, 2851

Carretta, E., \& Gratton, R. G. 1997, A\&AS, 121, 95 
Cayrel, R., Perrin, M.-N., Barbuy, B., \& Buser, R. 1991, A\&A, 247, 108

Cayrel, R., Faurobert-Scholl, M., Feautrier, N., Spielfieldel, A., \& Thévenin, F. 1996, A\&A, 312, 549

Coelho, P., Barbuy, B., Meléndez, J., Schiavon, R. P., \& Castilho, B. V. 2005, A\&A, 443, 735

Cram, L. 1999, Trans. IAU XXIIIB, ed. J. Andersen, 141

Davidge, T. 2000, ApJS, 126, 105

Davidge, T. 2001, AJ, 121, 3100

Davidge, T., Ledlow, M., \& Puxley, P. 2004, AJ, 128, 300

Dean, J. F., Warpen, P. R., \& Cousins, A. J. 1978, MNRAS, 183, 569

Dufay, J., Berthier, P., \& Morignat, B. 1954, Publications of the Observatoire de Haute-Provence, 3, No. 17

Edvardsson, B., Andersen, J., Gustafsson, B., et al. 1993, A\&A, 102, 603

Grevesse, N., \& Sauval, J. 1998, Space Sci. Rev., ed. S. S. Holt, \& G. Sonneborn (San Francisco: ASP), 85, 161

Gustafsson, B., Bell, R. A., Eriksson, K., \& Nordlund, Å. 1975, A\&A, 42,407

Harris, W. E. 1996, AJ, 112, 1487

Hinkle, K., Wallace, L., Valenti, J., \& Harmer, D. 2000, Visible and Near Infrared Atlas of the Arcturus Spectrum 3727-9300 ̊ (San Francisco: ASP)

Ivans, I., Sneden, C., Kraft, R. P., et al. 1999, AJ, 118, 1273

Landolt, A. 1992, AJ, 104, 340

Lawler, J. E., Wickliffe, M. E., den Hartog, E. A., \& Sneden, C. 2001a, ApJ, 563, 1075

Lawler, J. E., Bonvallet, G., \& Sneden, C. 2001b, ApJ, 556, 452

Martin, W. C., Fuhr, J. R., Kelleher, D. E., et al. 2002, NIST Atomic Database (version 2.0), http://physics.nist.gov/asd, National Institute of Standards and Technology, Gaithersburg, MD
Matteucci, F., \& Brocato, E. 1990, ApJ, 365, 539

Matteucci, F., Romano, D., \& Molaro, P. 1999, A\&A, 341, 458

McWilliam, A., \& Rich, R. M. 1994, ApJS, 91, 749

McWilliam, A., \& Rich, R. M. 2003, in Origin and Evolution of the Elements, http://www.ociw.edu/ociw/ symposia/series/symposium4/proceedings.html (MR03) Meléndez, J., Barbuy, B., Bica, E., et al. 2003, A\&A, 411, 417 Minniti, D. 1995, A\&A, 303, 468

Minniti, D., Olszewski, E. W., \& Rieke, M. 1995, AJ, 110, 1686

Momany, Y., Ortolani, S., Held, E. V., et al. 2003, A\&A, 402, 607

Origlia, L., \& Rich, R. M. 2004, AJ, 127, 3422

Origlia, L., Valenti, E., Rich, R. M., \& Ferraro, F. R. 2005, MNRAS, 363,897

Ortolani, S., Bica, E., \& Barbuy, B. 1997, MNRAS, 284, 692

Ortolani, S., Bica, E., \& Barbuy, B. 1999, A\&AS, 138, 267

Ortolani, S., Barbuy, B., Bica, E., et al. 2001, A\&A, 376, 878

Plez, B., Brett, J. M., \& Nordlund, Å. 1992, A\&A, 256, 551

Pompéia, L., Barbuy, B., \& Grenon, M. 2003, ApJ, 592, 1173

Ramírez, S. V., \& Cohen, J. 2002, AJ, 123, 3277

Rich, R. M., Sosin, C., Djorgovski, S. G., et al. 1997, ApJ, 484, L25

Rieke, G. H., \& Lebofsky, M. J. 1985, ApJ, 288, 618

Skrutskie, M., Schneider, S. E., Stiening, R., et al. 1997, in The Impact of Large Scale Near-IR Sky Surveys, ed. Garzon et al. (Netherlands: Kluwer), 210, 187

Spite, M. 1967, Ann. d'Astroph., 30, 211

Stetson, P. B. 1994, PASP, 106, 250

Stetson, P. B., \& Pancino, E. 2006, in preparation

Thévenin, F., \& Idiart, T. 1999, ApJ, 521, 753

Trager, S. C., King, I. R., \& Djorgovski, S. 1995, AJ, 109, 218

van den Bergh, S. 1993, ApJ, 411, 178

Zinn, R., \& West, M. J. 1984, ApJS, 55, 45

Zoccali, M., Barbuy, B., Hill, V., et al. 2004, A\&A, 423, 507 
B. Barbuy et al.: VLT-UVES analysis of two giants in the bulge metal-poor globular cluster HP-1, Online Material p 1

\section{Online Material}


B. Barbuy et al.: VLT-UVES analysis of two giants in the bulge metal-poor globular cluster HP-1, Online Material p 2

Table 6. Abundance ratios derived and atomic parameters adopted.

\begin{tabular}{|c|c|c|c|c|c|c|c|c|c|c|c|}
\hline \multirow{3}{*}{$\begin{array}{l}\text { Species } \\
{[\mathrm{OI}]}\end{array}$} & \multirow{3}{*}{$\begin{array}{l}\lambda \\
(\AA) \\
6300.311\end{array}$} & \multirow{3}{*}{$\begin{array}{l}\chi_{\mathrm{ex}} \\
(\mathrm{eV}) \\
0.00\end{array}$} & \multirow{3}{*}{$\begin{array}{l}C_{6} \\
0.30 \mathrm{E}-31\end{array}$} & \multicolumn{2}{|c|}{$\log g f$} & \multicolumn{3}{|c|}{$[\mathrm{X} / \mathrm{Fe}]$} & \multirow{2}{*}{\multicolumn{2}{|c|}{$\begin{array}{c}E W \quad(\mathrm{~m} \AA) \\
\text { HP1-2 } \\
\end{array}$}} & \multirow[b]{2}{*}{ HP1-3 } \\
\hline & & & & \multirow{2}{*}{$\begin{array}{r}\text { ZOC04 } \\
-9.716\end{array}$} & \multirow{2}{*}{$\begin{array}{r}\text { present } \\
-9.716\end{array}$} & \multirow{2}{*}{$\begin{array}{r}\text { Arcturus } \\
+0.20\end{array}$} & \multirow{2}{*}{$\begin{array}{r}\text { HP1-2 } \\
+0.4\end{array}$} & \multirow{2}{*}{$\begin{array}{r}\text { HP1-3 } \\
+0.4\end{array}$} & & & \\
\hline & & & & & & & & & 34.8 & 33.2 & \\
\hline $\mathrm{NaI}$ & 6154.230 & 2.10 & $0.90 \mathrm{E}-31$ & $-1.56^{1}$ & -1.56 & -0.2 & 0.0 & +0.0 & 19.0 & 33.8 & \\
\hline $\mathrm{NaI}$ & 6160.753 & 2.10 & $0.30 \mathrm{E}-31$ & $-1.26^{1}$ & -1.26 & -0.2 & 0.0 & +0.0 & 22.0 & - & \\
\hline $\mathrm{MgI}$ & 6318.720 & 5.11 & $0.30 \mathrm{E}-31$ & $-2.10^{2}$ & -2.10 & +0.30 & 0.0 & +0.20 & 16.2 & 32.4 & \\
\hline $\mathrm{MgI}$ & 6319.242 & 5.11 & $0.30 \mathrm{E}-31$ & $-2.36^{2}$ & -2.36 & +0.30 & 0.0 & +0.20 & 12.8 & 22.6 & \\
\hline $\mathrm{MgI}$ & 6319.490 & 5.11 & $0.30 \mathrm{E}-31$ & $-2.80^{2}$ & -2.90 & +0.30 & - & +0.20 & - & 3.6 & \\
\hline $\mathrm{MgI}$ & 6765.450 & 5.75 & $0.30 \mathrm{E}-31$ & $-1.94^{3}$ & -1.94 & +0.30 & 0.0 & +0.20 & 7.4 & 7.1 & \\
\hline SiI & 5948.548 & 5.08 & $2.19 \mathrm{E}-30^{a}$ & $-1.17^{2}$ & -1.17 & +0.25 & +0.3 & +0.3 & 80.8 & 58.2 & \\
\hline SiI & 6142.494 & 5.62 & $0.30 \mathrm{E}-31$ & $-1.50^{2}$ & -1.58 & +0.25 & +0.3 & +0.3 & 22.3 & 18.7 & \\
\hline SiI & 6145.020 & 5.61 & $0.30 \mathrm{E}-31$ & $-1.45^{2}$ & -1.5 & +0.25 & +0.3 & +0.3 & 21.0 & 20.0 & \\
\hline SiI & 6155.142 & 5.62 & $0.30 \mathrm{E}-30$ & $-0.85^{2}$ & -0.85 & +0.35 & +0.3 & +0.3 & 53.1 & 55.4 & \\
\hline SiI & 6237.328 & 5.61 & $0.30 \mathrm{E}-30$ & $-1.01^{3}$ & -1.1 & +0.25 & +0.2 & +0.3 & 29.6 & 39.0 & \\
\hline SiI & 6243.823 & 5.61 & $0.30 \mathrm{E}-32$ & $-1.30^{4}$ & -1.30 & +0.25 & - & +0.2 & - & 19.7 & \\
\hline SiI & 6414.987 & 5.87 & $0.30 \mathrm{E}-30$ & $-1.13^{2}$ & -1.13 & +0.35 & - & +0.3 & - & 21.7 & \\
\hline SiI & 6721.844 & 5.86 & $0.90 \mathrm{E}-30$ & $-1.17^{3}$ & -1.17 & +0.25 & +0.3 & +0.3 & 23.5 & 30.5 & \\
\hline $\mathrm{CaI}$ & 6102.727 & 1.88 & $4.54 \mathrm{E}-31^{a}$ & $-0.79^{1}$ & -0.93 & +0.25 & +0.1 & +0.1 & 137.7 & 143.8 & \\
\hline $\mathrm{CaI}$ & 6156.030 & 2.52 & $4.0 \mathrm{E}-31^{a a}$ & $-2.39^{2}$ & -2.59 & +0.20 & -0.1 & +0.2 & 2.3 & 14.5 & \\
\hline $\mathrm{CaI}$ & 6161.295 & 2.51 & $4.0 \mathrm{E}-31^{a a}$ & $-1.02^{1}$ & -1.42 & +0.10 & +0.0 & +0.1 & 37.2 & - & \\
\hline $\mathrm{CaI}$ & 6162.167 & 1.89 & $3.0 \mathrm{E}-31^{a a}$ & $-0.09^{1}$ & -0.09 & -0.10 & +0.0 & +0.1 & 200.0 & - & \\
\hline $\mathrm{CaI}$ & 6166.440 & 2.52 & $3.97 \mathrm{E}-31^{a a}$ & $-0.90^{1}$ & -1.156 & +0.20 & +0.0 & +0.1 & 78.2 & 104.2 & \\
\hline $\mathrm{CaI}$ & 6169.044 & 2.52 & $3.97 \mathrm{E}-31^{a a}$ & $-0.54^{1}$ & -0.90 & +0.10 & +0.0 & +0.1 & 72.6 & 105.4 & \\
\hline $\mathrm{CaI}$ & 6169.564 & 2.52 & $4.0 \mathrm{E}-31^{a a}$ & $-0.27^{1}$ & -0.63 & +0.10 & +0.0 & +0.1 & 98.0 & - & \\
\hline $\mathrm{CaI}$ & 6439.080 & 2.52 & $3.4 \mathrm{E}-32^{a a}$ & $+0.3^{4}$ & +0.3 & +0.00 & +0.0 & $+0.1:$ & 166.5 & - & \\
\hline $\mathrm{CaI}$ & 6455.605 & 2.52 & $3.39 \mathrm{E}-32^{a a}$ & $-1.35^{1}$ & -1.55 & +0.10 & $+0.0:$ & - & 55.2 & - & \\
\hline $\mathrm{CaI}$ & 6464.679 & 2.52 & $3.4 \mathrm{E}-32^{a a}$ & $-2.10^{2}$ & -2.48 & +0.40 & - & - & - & - & \\
\hline $\mathrm{CaI}$ & 6471.668 & 2.52 & $3.39 \mathrm{E}-32^{a a}$ & $-0.59^{1}$ & -0.80 & +0.00 & -0.1 & +0.1 & 74.0 & 95.6 & \\
\hline $\mathrm{CaI}$ & 6493.788 & 2.52 & $3.37 \mathrm{E}-32^{a a}$ & $+0.14^{1}$ & 0.0 & -0.15 & -0.1 & -0.1 & 141.0 & 146.9 & \\
\hline $\mathrm{CaI}$ & 6499.654 & 2.52 & $3.37 \mathrm{E}-32^{a a}$ & $-0.59^{1}$ & -0.85 & -0.15 & -0.1 & +0.1 & 81.0 & 110.9 & \\
\hline $\mathrm{CaI}$ & 6508.846 & 2.52 & $3.37 \mathrm{E}-32^{a a}$ & $-2.50^{3}$ & -2.51 & +0.10 & - & - & - & - & \\
\hline $\mathrm{CaI}$ & 6572.779 & 0.00 & $1.75 \mathrm{E}-32^{a a}$ & $-4.29^{1}$ & -4.32 & +0.10 & -0.1 & +0.1 & 63.4 & 111.3 & \\
\hline $\mathrm{CaI}$ & 6717.687 & 2.71 & 4.1E-31 $1^{a a}$ & $-0.61^{1}$ & -0.61 & +0.10 & -0.1 & +0.1 & - & 131.9 & \\
\hline TiI & 5866.452 & 1.07 & $1.44 \mathrm{E}-32^{a a}$ & $-0.84^{1}$ & -0.84 & +0.1 & +0.0 & +0.0 & 80.5 & 109.5 & \\
\hline TiI & 5922.110 & 1.05 & $2.32 \mathrm{E}-32^{a a}$ & $-1.47^{1}$ & -1.50 & +0.1 & +0.1 & +0.0 & 56.3 & 66.8 & \\
\hline TiI & 5965.835 & 1.88 & $2.14 \mathrm{E}-32^{a}$ & $-0.41^{1}$ & -0.45 & +0.1 & +0.1 & +0.0 & 48.4 & 77.3 & \\
\hline TiI & 5978.549 & 1.87 & $1.43 \mathrm{E}-32^{a a}$ & $-0.50^{1}$ & -0.55 & +0.1 & +0.1 & +0.0 & 27.4 & 52.4 & \\
\hline TiI & 6064.626 & 1.05 & $1.37 \mathrm{E}-32^{a a}$ & $-1.94^{1}$ & -1.94 & +0.1 & - & - & - & - & \\
\hline TiI & 6091.177 & 2.27 & $2.59 \mathrm{E}-32^{a a}$ & $-0.42^{1}$ & -0.42 & +0.0 & +0.1 & +0.0 & 18.1 & 45.7 & \\
\hline TiI & 6126.224 & 1.07 & $1.37 \mathrm{E}-32^{a a}$ & $-1.43^{1}$ & -1.43 & +0.0 & +0.0 & +0.0 & 50.0 & 68.3 & \\
\hline TiI & 6258.110 & 1.44 & $3.17 \mathrm{E}-32^{a a}$ & $-0.36^{1}$ & -0.36 & +0.1 & -0.1 & +0.0 & 63.5 & 113.2 & \\
\hline TiI & 6261.106 & 1.43 & $4.68 \mathrm{E}-32^{a}$ & $-0.48^{1}$ & -0.48 & +0.1 & +0.1 & +0.0 & 91.3 & 120.9 & \\
\hline TiI & 6303.767 & 1.44 & $3.12 \mathrm{E}-32^{a a}$ & $-1.57^{1}$ & -1.57 & +0.1 & +0.1 & +0.1 & 16.1 & 40.6 & \\
\hline TiI & 6312.238 & 1.46 & $3.17 \mathrm{E}-32^{a a}$ & $-1.69^{1}$ & -1.69 & +0.2 : & +0.1 & +0.0 & - & 31.2 & \\
\hline TiI & 6336.113 & 1.44 & $1.86 \mathrm{E}-32^{a a}$ & $-1.74^{1}$ & -1.74 & +0.1 & - & $+0.1:$ & 22.2 & 32.8 & \\
\hline TiI & 6508.154 & 1.43 & $1.46 \mathrm{E}-32^{a}$ & $-2.05^{3}$ & -2.05 & +0.1 & - & +0.0 & - & 10.9 & \\
\hline TiI & 6554.238 & 1.44 & $1.81 \mathrm{E}-32^{a a}$ & $-1.22^{1}$ & -1.22 & +0.1 & -0.1 & +0.0 & 22.7 & 67.3 & \\
\hline TiI & 6556.077 & 1.46 & $1.81 \mathrm{E}-32^{a a}$ & $-1.07^{1}$ & -1.07 & +0.1 & +0.1 & +0.0 & 41.3 & 68.6 & \\
\hline TiI & 6599.113 & 0.90 & $1.96 \mathrm{E}-32^{a a}$ & $-2.09^{1}$ & -2.09 & +0.1 & +0.1 & +0.0 & 20.8 & 55.1 & \\
\hline
\end{tabular}


B. Barbuy et al.: VLT-UVES analysis of two giants in the bulge metal-poor globular cluster HP-1, Online Material p 3

Table 6. continued.

\begin{tabular}{|c|c|c|c|c|c|c|c|c|c|c|c|}
\hline \multirow[t]{2}{*}{ Species } & \multirow{2}{*}{$\begin{array}{l}\lambda \\
(\AA)\end{array}$} & \multirow{2}{*}{$\begin{array}{l}\chi_{\mathrm{ex}} \\
(\mathrm{eV})\end{array}$} & \multirow[t]{2}{*}{$\overline{C_{6}}$} & \multicolumn{2}{|c|}{$\log g f$} & \multicolumn{3}{|c|}{$[\mathrm{X} / \mathrm{Fe}]$} & \multirow{2}{*}{\multicolumn{2}{|c|}{$\begin{array}{c}E W \quad(\mathrm{~m} \AA) \\
\text { HP1-2 }\end{array}$}} & \multirow[b]{2}{*}{ HP1-3 } \\
\hline & & & & ZOC04 & present & Arcturus & HP1-2 & HP1-3 & & & \\
\hline TiI & 6743.127 & 0.90 & $0.30 \mathrm{E}-31$ & $-1.63^{1}$ & -1.73 & +0.1 & -0.2 & +0.0 & 32.9 & 79.7 & \\
\hline TilI & 6491.580 & 2.06 & $0.30 \mathrm{E}-31$ & $-2.10^{1 *}$ & -2.10 & +0.1 & +0.1 & $+0.3:$ & 73.2 & 79.1 & \\
\hline TiII & 6559.576 & 2.05 & $0.30 \mathrm{E}-31$ & $-2.48^{3}$ & -2.35 & +0.1 & - & +0.1 & - & 5.4 & \\
\hline TiII & 6606.970 & 2.06 & $0.30 \mathrm{E}-31$ & $-2.79^{1}$ & -2.85 & +0.1 & +0.1 & +0.0 & 22.9 & 22.9 & \\
\hline BaII & 6141.727 & 0.70 & & hfs & hfs & -0.20 & +0.2 & +0.2 & 158.1 & 162.1 & \\
\hline BaII & 6496.908 & 0.60 & & hfs & hfs & -0.20 & +0.0 & +0.2 & 192.0 & 179.6 & \\
\hline LaII & 6390.480 & 0.32 & & hfs & hfs & -0.30 & 0.0 & +0.0 & 18.0 & 20.3 & \\
\hline EuII & 6645.127 & 1.38 & & hfs & hfs & +0.30 & +0.0 & +0.3 & 17.4 & 31.9 & \\
\hline
\end{tabular}

${ }^{a}$ Damping constants computed following Barklem et al. (1998, 2000); ${ }^{a}$ damping constants computed as in ${ }^{a}$, decreased by a factor $1.5\left(C_{6} / 1.5\right)$. Other $C_{6}$ values are van der Waals classical ones, as described in Zoccali et al. (2004). Column 5 reports the log $g f$ used in Zoccali et al. (2004), adopted from ${ }^{1}$ NIST/VALD; ${ }^{2}$ Barbuy et al. (1999); ${ }^{3}$ McWilliam \& Rich (1994); ${ }^{4}$ Bensby et al. (2004), whereas Col. 6 gives the values derived in this work from a fit to the solar spectrum; * for TiII $6491.58 \AA$ line, the NIST $\log g f=-2.10$ is different from the VALD value of $\log g f=-1.793$. 
B. Barbuy et al.: VLT-UVES analysis of two giants in the bulge metal-poor globular cluster HP-1, Online Material p 4

\section{Appendix A}

Table A.1. Fe I and Fe II line list, wavelength, excitation potential, damping constant, and $g f$-values. For HP1-3, Equivalent widths were measured using both DAOSPEC and line-by-line with IRAF.

\begin{tabular}{|c|c|c|c|c|c|c|}
\hline Species & $\lambda(\AA)$ & $\chi_{\mathrm{ex}}(\mathrm{eV})$ & $C_{6}$ & $\log g f$ & HP1-2(EW) & HP1-3(EW) \\
\hline Fe II & 6084.12 & 3.20 & $3.0 \mathrm{E}-32$ & -3.81 & 24.5 & 13.1 \\
\hline $\mathrm{Fe}$ II & 6149.19 & 3.89 & $3.0 \mathrm{E}-32$ & -2.31 & - & 27.0 \\
\hline Fe II & 6247.59 & 3.89 & $3.0 \mathrm{E}-32$ & -2.33 & - & 44.7 \\
\hline Fe II & 6432.68 & 2.89 & $3.0 \mathrm{E}-32$ & -3.61 & 49.5 & 34.3 \\
\hline $\mathrm{Fe}$ II & 6456.38 & 3.90 & $3.0 \mathrm{E}-32$ & -2.08 & 69.7 & 53.8 \\
\hline $\mathrm{Fe} \mathrm{I}$ & 6065.48 & 2.61 & $1.5 \mathrm{E}-32$ & -1.53 & - & 137.6 \\
\hline $\mathrm{Fe} \mathrm{I}$ & 6079.01 & 4.65 & $1.6 \mathrm{E}-31$ & -1.126 & - & 27.9 \\
\hline $\mathrm{Fe} \mathrm{I}$ & 6082.71 & 2.22 & $1.0 \mathrm{E}-32$ & -3.572 & - & 62.7 \\
\hline $\mathrm{Fe} \mathrm{I}$ & 6085.26 & 2.76 & $1.4 \mathrm{E}-32$ & -3.214 & 54.7 & 99.5 \\
\hline $\mathrm{Fe} \mathrm{I}$ & 6093.64 & 4.61 & $3.0 \mathrm{E}-32$ & -1.449 & - & 99.5 \\
\hline Fe I & 6096.67 & 3.98 & $1.8 \mathrm{E}-31$ & -1.931 & - & 40.2 \\
\hline $\mathrm{Fe} \mathrm{I}$ & 6137.00 & 2.20 & $8.5 \mathrm{E}-33$ & -2.950 & 113.8 & 111.2 \\
\hline $\mathrm{Fe} \mathrm{I}$ & 6151.62 & 2.18 & $8.1 \mathrm{E}-33$ & -3.299 & 72.3 & - \\
\hline $\mathrm{Fe} \mathrm{I}$ & 6157.73 & 4.08 & $7.1 \mathrm{E}-32$ & -1.249 & 59.3 & 62.1 \\
\hline $\mathrm{Fe} \mathrm{I}$ & 6159.38 & 4.61 & $1.3 \mathrm{E}-31$ & -1.968 & - & 12.8 \\
\hline $\mathrm{Fe} \mathrm{I}$ & 6165.36 & 4.14 & $7.7 \mathrm{E}-32$ & -1.549 & - & 38.9 \\
\hline $\mathrm{Fe} \mathrm{I}$ & 6170.51 & 4.80 & $2.3 \mathrm{E}-31$ & -0.430 & 59.1 & - \\
\hline $\mathrm{Fe} \mathrm{I}$ & 6173.34 & 2.22 & $8.4 \mathrm{E}-33$ & -2.879 & 116.2 & 97.9 \\
\hline $\mathrm{Fe} \mathrm{I}$ & 6180.20 & 2.73 & $1.3 \mathrm{E}-32$ & -2.784 & 76.4 & 77.7 \\
\hline $\mathrm{Fe} \mathrm{I}$ & 6187.99 & 3.94 & $1.6 \mathrm{E}-31$ & -1.718 & 47.2 & - \\
\hline $\mathrm{Fe} I$ & 6200.31 & 2.61 & $1.5 \mathrm{E}-32$ & -2.437 & 99.0 & - \\
\hline $\mathrm{Fe} \mathrm{I}$ & 6213.43 & 2.22 & $8.4 \mathrm{E}-33$ & -2.646 & 118.0 & 111.7 \\
\hline $\mathrm{Fe} \mathrm{I}$ & 6215.14 & 4.19 & $7.9 \mathrm{E}-32$ & -1.438 & 46.2 & 51.8 \\
\hline $\mathrm{Fe} \mathrm{I}$ & 6219.28 & 2.20 & $8.2 \mathrm{E}-33$ & -2.434 & 124.8 & - \\
\hline $\mathrm{Fe} \mathrm{I}$ & 6220.78 & 3.88 & $1.3 \mathrm{E}-31$ & -2.462 & - & 6.3 \\
\hline $\mathrm{Fe} \mathrm{I}$ & 6226.74 & 3.88 & $1.3 \mathrm{E}-31$ & -2.202 & 15.8 & 34.7 \\
\hline $\mathrm{Fe} \mathrm{I}$ & 6229.23 & 2.85 & $1.5 \mathrm{E}-32$ & -2.973 & 38.6 & 58.8 \\
\hline Fe I & 6240.65 & 2.22 & $1.4 \mathrm{E}-32$ & -3.388 & 72.5 & 90.1 \\
\hline $\mathrm{Fe} \mathrm{I}$ & 6246.32 & 3.60 & $1.2 \mathrm{E}-31$ & -0.956 & 110.0 & 107.6 \\
\hline $\mathrm{Fe} \mathrm{I}$ & 6256.36 & 2.45 & $1.3 \mathrm{E}-32$ & -2.624 & 123.4 & - \\
\hline $\mathrm{Fe} \mathrm{I}$ & 6265.13 & 2.18 & $7.9 \mathrm{E}-33$ & -2.550 & - & 131.0 \\
\hline $\mathrm{Fe} \mathrm{I}$ & 6270.23 & 2.86 & $1.5 \mathrm{E}-32$ & -2.711 & - & 71.1 \\
\hline $\mathrm{Fe} \mathrm{I}$ & 6271.28 & 3.33 & $8.9 \mathrm{E}-32$ & -2.957 & 20.8 & 35.2 \\
\hline Fe I & 6297.79 & 2.22 & $8.2 \mathrm{E}-33$ & -2.740 & 103.0 & 101.6 \\
\hline $\mathrm{Fe} \mathrm{I}$ & 6311.50 & 2.83 & $1.4 \mathrm{E}-32$ & -3.224 & 38.6 & 44.2 \\
\hline $\mathrm{Fe} I$ & 6315.81 & 4.08 & $6.6 \mathrm{E}-32$ & -1.712 & 33.6 & 43.1 \\
\hline $\mathrm{Fe} \mathrm{I}$ & 6322.69 & 2.59 & $1.4 \mathrm{E}-32$ & -2.426 & 96.8 & - \\
\hline $\mathrm{Fe} \mathrm{I}$ & 6330.85 & 4.73 & $1.6 \mathrm{E}-31$ & -1.737 & - & 14.5 \\
\hline $\mathrm{Fe} \mathrm{I}$ & 6335.33 & 2.20 & $8.0 \mathrm{E}-33$ & -2.229 & 134.8 & - \\
\hline $\mathrm{Fe} \mathrm{I}$ & 6336.82 & 3.69 & $1.3 \mathrm{E}-31$ & -1.053 & 113.8 & - \\
\hline $\mathrm{Fe} \mathrm{I}$ & 6344.15 & 2.43 & $1.2 \mathrm{E}-32$ & -2.922 & 80.5 & 94.1 \\
\hline $\mathrm{Fe} \mathrm{I}$ & 6355.03 & 2.85 & $1.4 \mathrm{E}-32$ & -2.405 & 95.5 & - \\
\hline Fe I & 6362.88 & 4.19 & $7.4 \mathrm{E}-32$ & -1.974 & 36.7 & - \\
\hline $\mathrm{Fe} \mathrm{I}$ & 6385.72 & 4.73 & $1.5 \mathrm{E}-31$ & -1.906 & 10.4 & 6.2 \\
\hline $\mathrm{Fe} \mathrm{I}$ & 6392.54 & 2.28 & $1.1 \mathrm{E}-32$ & -4.028 & 31.9 & - \\
\hline
\end{tabular}


B. Barbuy et al.: VLT-UVES analysis of two giants in the bulge metal-poor globular cluster HP-1, Online Material p 5

Table A.1. continued.

\begin{tabular}{lcrrrrr}
\hline \hline Species & $\lambda(\AA)$ & $\chi_{\text {ex }}(\mathrm{eV})$ & $C_{6}$ & $\log g f$ & $\mathrm{HP} 1-2(E W)$ & $\mathrm{HP} 1-3(E W)$ \\
\hline Fe I & 6411.65 & 3.65 & $1.2 \mathrm{E}-31$ & -0.821 & 58.7 & 120.8 \\
Fe I & 6419.95 & 4.73 & $1.5 \mathrm{E}-31$ & -0.250 & 55.2 & 67.2 \\
Fe I & 6469.19 & 4.84 & $1.9 \mathrm{E}-31$ & -0.771 & 27.5 & - \\
Fe I & 6475.62 & 2.56 & $1.5 \mathrm{E}-32$ & -2.941 & 71.9 & - \\
Fe I & 6481.87 & 2.28 & $1.1 \mathrm{E}-32$ & -2.984 & 99.1 & - \\
Fe I & 6496.47 & 4.80 & $1.7 \mathrm{E}-31$ & -0.570 & - & 49.6 \\
Fe I & 6518.37 & 2.83 & $1.3 \mathrm{E}-32$ & -2.748 & - & 82.7 \\
Fe I & 6546.24 & 2.76 & $1.2 \mathrm{E}-32$ & -1.648 & 130.3 & - \\
Fe I & 6569.22 & 4.73 & $1.3 \mathrm{E}-31$ & -0.422 & 61.0 & 63.9 \\
Fe I & 6575.02 & 2.59 & $1.4 \mathrm{E}-32$ & -2.824 & 82.1 & - \\
Fe I & 6593.87 & 2.43 & $1.1 \mathrm{E}-32$ & -2.422 & 123.3 & 112.4 \\
Fe I & 6597.56 & 4.80 & $1.2 \mathrm{E}-32$ & -1.061 & - & 26.9 \\
Fe I & 6608.03 & 2.28 & $1.0 \mathrm{E}-32$ & -4.038 & - & 35.5 \\
Fe I & 6609.11 & 2.56 & $1.3 \mathrm{E}-32$ & -2.692 & 77.9 & 83.7 \\
Fe I & 6627.54 & 4.55 & $9.9 \mathrm{E}-32$ & -1.683 & - & 22.0 \\
Fe I & 6633.41 & 4.84 & $1.7 \mathrm{E}-31$ & -1.490 & - & 13.5 \\
Fe I & 6633.75 & 4.56 & $1.4 \mathrm{E}-31$ & -0.779 & - & 49.7 \\
Fe I & 6634.11 & 4.80 & $1.5 \mathrm{E}-31$ & -1.432 & - & 23.3 \\
Fe I & 6639.88 & 4.08 & $5.7 \mathrm{E}-32$ & -2.462 & 10.7 & - \\
Fe I & 6646.93 & 2.61 & $1.4 \mathrm{E}-32$ & -3.991 & - & 18.8 \\
Fe I & 6653.85 & 4.15 & $1.8 \mathrm{E}-31$ & -2.520 & - & 8.9 \\
Fe I & 6663.44 & 2.42 & $1.3 \mathrm{E}-32$ & -2.478 & 122.6 & - \\
\hline & & & & & &
\end{tabular}

